\title{
MISpherolD: a knowledgebase and transparency tool for minimum information in spheroid identity
}

\begin{abstract}
Arne Peirsman $\left({ }^{1,2,3,28}\right.$, Eva Blondeel 1 1,2,28, Tasdiq Ahmed ${ }^{4}{ }^{4}$, Jasper Anckaert ${ }^{5,6}$, Dominique Audenaert ${ }^{7}$, Tom Boterberg ${ }^{8}$, Krisztina Buzas ${ }^{9}$, Neil Carragher ${ }^{10}{ }^{10}$, Gastone Castellani ${ }^{11}$, Flávia Castro ${ }^{12}$, Virginie Dangles-Marie ${ }^{13,14}$, John Dawson ${ }^{10}$, Pascal De Tullio ${ }^{15}$, Elly De Vlieghere $\mathbb{1}^{1,2}$, Sándor Dedeyne ${ }^{1,2}$, Herman Depypere ${ }^{16}$, Akos Diosdi ${ }^{17}$, Ruslan I. Dmitriev ${ }^{18}$, Helmut Dolznig ${ }^{10}{ }^{19}$, Suzanne Fischer ${ }^{1,2}$, Christian Gespach ${ }^{20}$, Vera Goossens ${ }^{7}$, Jyrki Heino ${ }^{21}$, An Hendrix ${ }^{1,2}$, Peter Horvath"17, Leoni A. Kunz-Schughart ${ }^{22}$, Sebastiaan Maes ${ }^{3}$, Christophe Mangodt ${ }^{1,2}$, Pieter Mestdagh $\mathbb{1}^{5,6}$, Soňa Michlíková ${ }^{22}{ }^{22}$, Maria José Oliveira ${ }^{12}$, Francesco Pampaloni ${ }^{23}$, Filippo Piccinini (iD) 24, Cláudio Pinheiro ${ }^{1,2}$, Jennifer Rahn ${ }^{25}$, Stephen M. Robbins ${ }^{25}$, Elina Siljamäki ${ }^{21}$, Patrick Steigemann ${ }^{26}$, Gwen Sys ${ }^{27}$, Shuichi Takayama ${ }^{4}$, Anna Tesei ${ }^{24}$, Joeri Tulkens ${ }^{1,2}$, Michiel Van Waeyenberge ${ }^{3}$, Jo Vandesompele ${ }^{10,6}$, Glenn Wagemans ${ }^{1,2}$, Claudia Weindorfer ${ }^{19}$, Nurten Yigit $\mathbb{1}^{5,6}$, Nina Zablowsky ${ }^{26}$, Michele Zanoni ${ }^{24}$, Phillip Blondeel ${ }^{3}$ and Olivier De Wever (1) ${ }^{1,2} \bowtie$

Spheroids are three-dimensional cellular models with widespread basic and translational application across academia and industry. However, methodological transparency and guidelines for spheroid research have not yet been established. The MISpheroID Consortium developed a crowdsourcing knowledgebase that assembles the experimental parameters of 3,058 published spheroid-related experiments. Interrogation of this knowledgebase identified heterogeneity in the methodological setup of spheroids. Empirical evaluation and interlaboratory validation of selected variations in spheroid methodology revealed diverse impacts on spheroid metrics. To facilitate interpretation, stimulate transparency and increase awareness, the Consortium defines the MISpherolD string, a minimum set of experimental parameters required to report spheroid research. Thus, MISpheroID combines a valuable resource and a tool for three-dimensional cellular models to mine experimental parameters and to improve reproducibility.
\end{abstract}

S pheroids, which are near-spherical multicellular aggregates, are one of the most common types of three-dimensional (3D) cell cultures. In contrast to two-dimensional (2D) cell cultures, spheroids have the advantage of maintaining a diffusive nutrient and oxygen supply, leading to metabolic gradients from the periphery to the inner core and causing spatial heterogeneity in proliferation, quiescence, necrosis and differentiation ${ }^{1,2}$. Spheroids are used as simplified biomimetic in vitro models to study fundamental

\footnotetext{
'Laboratory of Experimental Cancer Research, Cancer Research Institute, Ghent, Belgium. '2Department of Human Structure and Repair, Ghent University, Ghent, Belgium. ${ }^{3}$ Plastic, Reconstructive and Aesthetic Surgery, Ghent University Hospital, Ghent, Belgium. ${ }^{4}$ Wallace H Coulter Department of Biomedical Engineering and Petit Institute for Bioengineering and Bioscience, Georgia Institute of Technology and Emory School of Medicine, Atlanta, GA, USA. ${ }^{5}$ OncoRNALab, Cancer Research Institute, Ghent, Belgium. ${ }^{6}$ Department of Biomolecular Medicine, Ghent University, Ghent, Belgium. ${ }^{7} \mathrm{VIB}$ Screening Core and Ghent University Expertise Centre for Bioassay Development and Screening (C-BIOS-VIB), Ghent University, Ghent, Belgium. ${ }^{8}$ Department of Radiation Oncology, Ghent University Hospital, Ghent, Belgium. 'Department of Immunology, University of Szeged, Faculty of Medicine-Faculty of Science and Informatics, Szeged, Hungary. ${ }^{10}$ Institute of Genetics and Cancer, Cancer Research UK Edinburgh Centre, University of Edinburgh, Edinburgh, UK. "Department of Experimental, Diagnostic and Specialty Medicine, University of Bologna, Bologna, Italy. ${ }^{12} \mathrm{i} 3 \mathrm{~S}$ - Institute for Research and Innovation in Health, University of Porto, Porto, Portugal. ${ }^{13}$ Translational Research Department, Institut Curie, PSL Research University, and Faculty of Pharmacy, Paris, France. ${ }^{14}$ Faculty of Pharmacy, Université Paris Descartes, Paris, France. ${ }^{15}$ Center for Interdisciplinary Research on Medicines (CIRM), Metabolomics Group, Université de Liège, Liège, Belgium. ${ }^{16}$ Menopause and Breast Clinic, Ghent University Hospital, Ghent, Belgium. ${ }^{17}$ Synthetic and Systems Biology Unit, Hungarian Academy of Sciences, Biological Research Center (BRC), Szeged, Hungary. ${ }^{18}$ Tissue Engineering and Biomaterials Group, Department of Human Structure and Repair, Ghent University, Ghent, Belgium. ${ }^{19}$ Institute of Medical Genetics, Medical University of Vienna, Vienna, Austria. ${ }^{20}$ INSERM U938 Hospital Saint-Antoine Research Center CRSA, Team Céline Prunier, TGFbeta Signaling in Cellular Plasticity and Cancer, Sorbonne University, Paris, France. ${ }^{21}$ Department of Life Technologies, University of Turku, Turku, Finland. ${ }^{22}$ OncoRay - National Center for Radiation Research in Oncology, University Hospital Carl Gustav Carus Dresden, Carl Gustav Carus Faculty of Medicine at TU Dresden, and Helmholtz-Zentrum Dresden-Rossendorf, Dresden, Germany. ${ }^{23}$ Physical Biology Group, Buchmann Institute for Molecular Life Sciences (BMLS), Goethe Universität Frankfurt am Main, Frankfurt am Main, Germany. ${ }^{24}$ IRCCS Istituto Romagnolo per lo Studio dei Tumori (IRST) 'Dino Amadori', Meldola, Italy. ${ }^{25}$ Departments of Oncology and Biochemistry and Molecular Biology, Cumming School of Medicine, University of Calgary, Calgary, Alberta, Canada. ${ }^{26}$ Lead Discovery, Nuvisan ICB, Berlin, Germany. ${ }^{27}$ Department of Orthopedics and Traumatology, Ghent University Hospital, Ghent University, Ghent, Belgium. ${ }^{28}$ These authors contributed equally: Arne Peirsman, Eva Blondeel. 凶e-mail: olivier.dewever@ugent.be
} 
mechanisms in biology and can be generated from a variety of cell cultures from healthy as well as pathological tissue, including cancer. Their scalability has promoted academic and industrial interest, particularly in the evaluation of drug responses or the biofabrication of $3 \mathrm{D}$ functional tissues or organs $\mathrm{s}^{3-8}$. The scientific literature uses several alternative terms, such as spheres, tumor(o)spheres and mammospheres, with each having a different definition'. To avoid confusion, the general term 'spheroid' will be used, to cover all aforementioned terms.

Spheroid production is based on the principle of self-assembly, which occurs when cells present in a non-adherent environment aggregate together. To induce these cellular interactions, numerous spheroid formation methods have been established such as spinner flasks, hanging drop cultures, microfluidic devices, cultures on low-adhesive substrates and so on $^{3}$. Spheroid biology, including cellular interactions and cell death, severely affect drug responsiveness ${ }^{10,11}$. Specific changes in methodological setup, such as the nutrient composition of cell culture media and the choice of spheroid formation method, may also contribute to differences in spheroid metrics $^{12-16}$. Although there is no one-size-fits-all methodological setup for spheroid experiments, it is currently unclear how heterogeneity in methodology affects spheroid metrics. Consequently, the usage of a diverse set of experimental settings requires transparent reporting, without which results are difficult to interpret, compare and reproduce ${ }^{17,18}$.

Despite decades of implementation of spheroid technology in various fields of life science and medical research ${ }^{19,20}$, no minimum information (MI) guidelines are available to cope with heterogeneity and encourage transparency. Minimum Information for Biological and Biomedical Investigations (MIBBI) provides access to the Minimum Information About a Cellular Assay (MIACA) and Minimum Information About Cell Migration Experiments (MIACME) guidelines, but these resources are not specific nor sufficient for spheroid experimentation given that they do not cope with the complexity of a $3 \mathrm{D}$ experiment ${ }^{21-23}$. This situation is in contrast to that for other biological fields, in which minimum information initiatives are available to define field-specific biological and technical parameters ${ }^{24-26}$.

To cope with this unmet need, we assembled an international consortium to develop the MISpheroID knowledgebase (https:// www.mispheroid.org). In-depth empirical evaluation and interlaboratory validation of selected variations in methodological setup identified a significant impact on a diverse set of spheroid metrics, while interrogation identified heterogeneity and a lack of transparency in published spheroid-related experiments. These results are merged into the generation of a minimum information string for spheroid interpretation. Thus, MISpheroID is a unique open-access resource that facilitates systematic reporting on essential spheroid methodology with the aim to increase consistency and awareness in both academic and industrial research environments.

\section{Results}

Creation of the MISpheroID knowledgebase. An initial literature screening identified spheroids derived from breast cancer cells as the most reported in the past decade (Supplementary Table 1). Consequently, we first conducted an in-depth methodological analysis of 1,628 breast cancer spheroid-related experiments, of which 1,506 were of human and 122 were of animal origin (Supplementary Fig. 1). For each experiment, we completed a checklist of 98 parameters relating to spheroid setup, characterization and application (Supplementary Table 2). Next, spheroid-related experiments from other tumor sites including the brain $(n=248)$, colorectum $(n=324)$, liver $(n=211)$, lung $(n=213)$, ovary $(n=243)$ and pancreas $(n=191)$ were evaluated, which resulted in an additional set of 1,430 experiments. Data were curated before inclusion in the
MISpheroID knowledgebase, which to date includes a total of 3,058 experiment entries.

Spheroid research practices. To identify practices in spheroid research, we performed an in-depth analysis of the MISpheroID knowledgebase. This found that 1,333 (of 1628, 81.9\%) unique protocols have been reported to establish and characterize breast cancer spheroids (Supplementary Fig. 2). Visualization of MISpheroID data shows inconsistent reporting and/or heterogeneity in breast cancer spheroid setup, characterization and application (Fig. 1). Culture medium type is not reported in $10 \%$ of experiments. In $47.5 \%$ of experiments the glucose concentration is not disclosed. This discrepancy probably results from the availability of $5.6 \mathrm{mM}$ (low glucose, LG) and $25 \mathrm{mM}$ (high glucose, HG) glucose-containing Dulbecco's Modified Eagle's Medium (DMEM) formulations and the ambiguous use of the term 'DMEM' to include all formulations of the medium. The spheroid formation method is efficiently reported $(97.3 \%)$, with liquid overlay as the most frequently applied method (71.1\%, Supplementary Fig. 3). Of these experiments, $52 \%$ use ultra-low attachment (ULA) plates and $42.1 \%$ use in situ coated plates. Agarose and poly-HEMA $(46.9 \%$ and $28.8 \%$, respectively) are the most implemented in situ coats. MISpheroID identifies 79 unique breast or mammary gland cell lines, with the estrogen-dependent MCF7 and T47D (542 (33.3\%) and $96(5.7 \%)$ out of 1,628 experiments, respectively) and the triple-negative MDAMB231 and 4T1 $337(20.7 \%)$ and 63 (3.8\%) out of 1,628 experiments, respectively) as the most frequently used breast or mammary gland cancer cell lines (Supplementary Fig. 4 and Supplementary Table 3). For each of these cell lines, a diverse set of culture media and formation methods is used to establish and study spheroids, although both variables are known to affect spheroid metrics ${ }^{11,12,14-16,27-30}$ (Extended Data Fig. 1 and Supplementary Fig. 5).

Although $87.8 \%$ of the breast cancer spheroid experiments are characterized by microscopy-based techniques, only $23.3 \%$ provide information about spheroid morphology. Despite the potential influence of spheroid size on study conclusions ${ }^{10,11}$, the numerical reporting of spheroid diameter (size), volume and projected area is described only in $21.0 \%, 4.5 \%$ and $2.9 \%$ of experiments, respectively. Shape assessment (for example, circularity) is performed in less than $1 \%$ of experiments. Characterization by non-microscopy-based techniques is mainly focused on RNA (for example, quantitative polymerase chain reaction, qPCR) and protein (for example, western blot) analysis in $26.6 \%$ and $25.9 \%$ of experiments, respectively (Fig. 1).

Spheroid application methods show that $23.9 \%$ of experiments focus on cancer stem cells, given that spheroid cultures from specific cell lines can be applied for cancer stem cell enrichment ${ }^{14}$. Functional assessments, such as migration and matrix invasion, are applied in $21.4 \%$ of experiments. Studies focused on pathophysiology research and drug testing (48.3\% and 24.6\%) vastly outnumber those on spheroid 3D culture optimization $(18.4 \%)$ (Supplementary Fig. 6).

In summary, breast or mammary gland cancer spheroid practices involve heterogeneity and/or lack of reporting in culture medium, spheroid formation method and spheroid size. Evaluation of these parameters for spheroid experiments from other tumor types indicates that lack of reporting in culture medium and spheroid size is widespread in the spheroid research field (Fig. 2). Critically, detailed evaluation of the reported medium types and spheroid formation method for the most frequently used cell line from each tumor type reveals an extensive heterogeneity (Extended Data Fig. 2, Supplementary Figs. 7 and 8 and Supplementary Table 3).

Impact of methodological heterogeneity on spheroid metrics. The heterogeneity and reporting deficiencies exposed by 
Parameter

Year of publication

$\%$

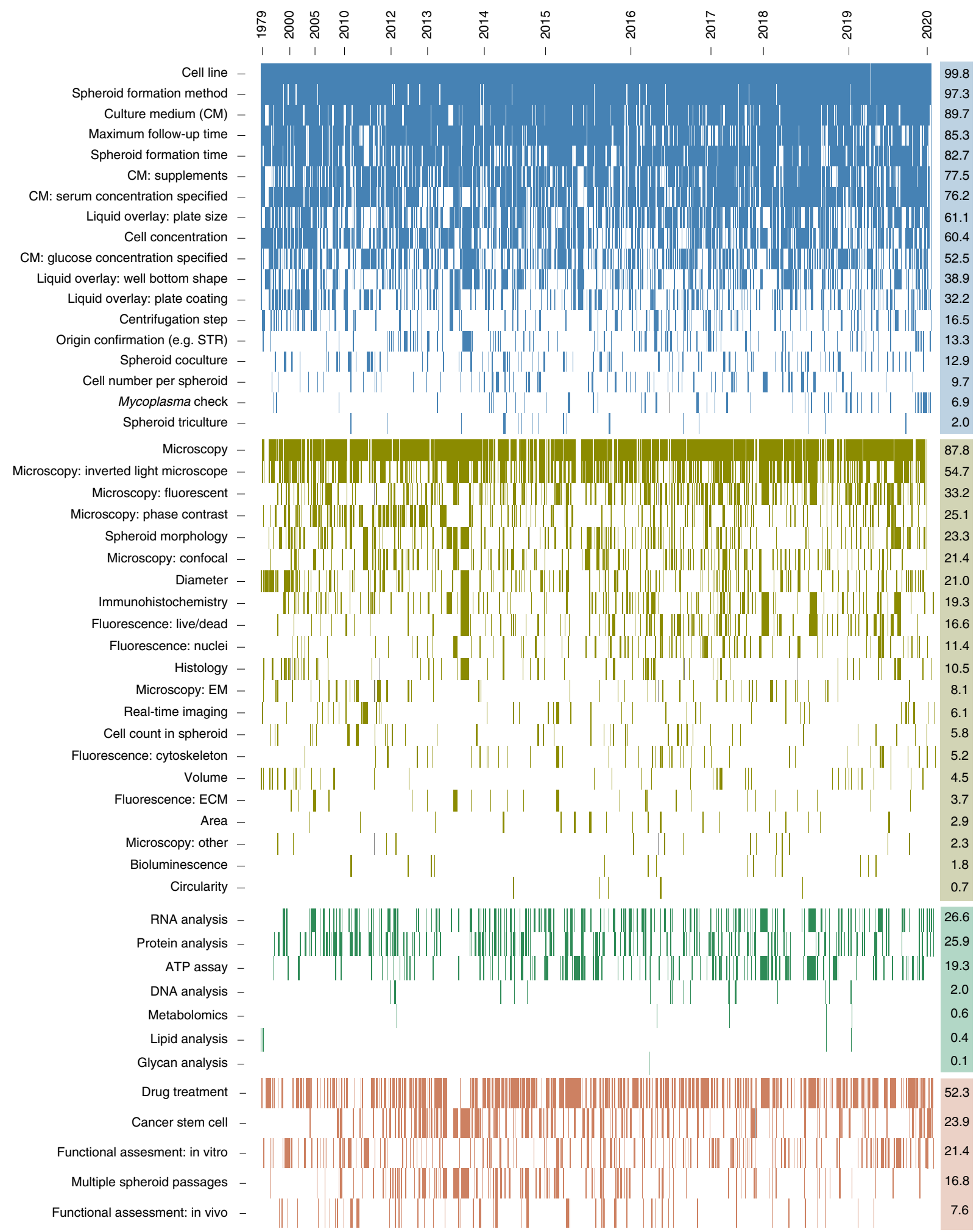

Fig. 1 | Mapping the reporting topography in breast cancer spheroid research. Binary heatmap showing the experimental parameters (rows, 51 of 98 parameters, selected for relevance) of each spheroid experiment (columns, $n=1,628$ ). The heatmap is divided vertically into three sections of parameters ('setup', 'characterization both microscopic and non-microscopic' and 'application'; indicated in blue, light and dark green, and red; and including 18, $21+7$, and 5 parameters, respectively) and horizontally according to the year of publication. For each section, rows are sorted in descending order according to total number of reported experimental parameters. Parameters that were not reported in an experiment appear as a white space in its corresponding column. The reporting efficiency of each parameter is indicated as a percentage in the right column. EM, electron microscopy; ECM, extracellular matrix. 


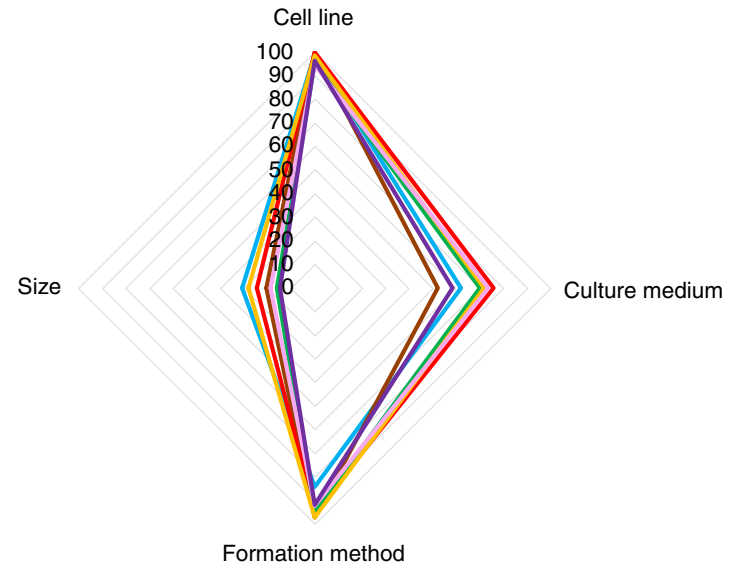

Formation method

- Brain - Breast - Colorectum - Liver - Lung - Ovary - Pancreas

Fig. 2 | Reporting efficiency of experimental parameters in spheroids from different tumor types. Spider plot visualization of the reporting percentage of cell line, culture medium, formation method and size in spheroids from different tumor types (clockwise). Axes represent the percentage of reporting efficiency.

MISpheroID interrogation prompted us to empirically evaluate the impact of culture medium type, spheroid formation method and spheroid size on complementary spheroid metrics using established cell lines and early passage patient-derived cell cultures from different tumor types.

Culture medium types commonly reported in the MISpheroID knowledgebase are DMEM (27.3\%), DMEM/F12 (25.6\%), RPMI1640 (18.1\%), Minimum Essential Medium (MEM) (3.5\%) and Eagle's Minimum Essential Medium (EMEM) (0.6\%), with each medium type having a different nutrient formulation (Extended Data Fig. 3). We measured media-induced transcriptional variation in lung cancer (A549), colorectal cancer (HCT116), ovarian cancer (SKOV3) and glioblastoma (U87MG) spheroids using RNA sequencing (RNA-seq). Principal component analysis showed differential variation in transcriptional response to culture media, with A549 and SKOV3 having the largest variation (Fig. 3a). For A549 the media separated into two clusters (RPMI1640, EMEM and MEM versus DMEM/F12, DMEMLG and DMEM HG), while for U87MG the variation was less striking but was still apparent, with DMEMHG and DMEMLG in one cluster and the other medium types in a second cluster.

Gene set enrichment analysis (GSEA) using the 50 hallmark gene sets $^{27}$ showed that the differentially expressed genes significantly converged on important hallmarks, and had medium-specific and cell type-specific patterns (Fig. 3b). In agreement with the principal component analyses, A549 and SKOV3 had the largest number of significantly enriched hallmark gene sets (37 and 38 of the 50 analyzed, respectively). The hallmark gene set analysis revealed a unique distinction between medium types within each cell type. While some medium types in the A549 analysis (EMEM and MEM) and in the U87MG analysis (DMEM HG) showed an enrichment of all hallmark signatures, other conditions showed discrete and unique diversities. The largest difference in the enrichment of hallmark gene sets between two medium types was observed for A549, DMEM/F12 versus MEM (mean difference, 1.90), for HCT116, DMEM/F12 versus MEM (mean difference, 1.08), for SKOV3, RPMI1640 versus MEM (mean difference, 1.09), and for U87MG, DMEMHG versus EMEM (mean difference, 2.45) (Supplementary Table 4).
To assess whether the medium-induced transcriptional changes are indicative of distinct cellular properties we examined spheroid metrics including cell death, adenosine triphosphate (ATP) content, ratio of lactate secretion to glucose uptake, secreted protein signatures of angiogenesis and immune interaction, circularity, size, and response to a cancer treatment intervention. These metrics were evaluated in the cell lines covered in the RNA-seq experiments in addition to spheroids derived from tumor types such as liver (HEPG2), human breast (MCF7), pancreas (PANC1), mouse mammary gland (4T1) and sarcoma (early passage patient-derived cultures SAR030, SAR120 and SAR121). Metrics were visualized as a spider plot (as a transformed Z-score), allowing direct comparison of the contribution of each medium for each spheroid type (Fig. 4a). All spheroid metrics were affected by medium type in both established and early passage cell cultures. Medium-induced changes were shared by some of the cell cultures examined while others were cell type specific, underscoring how the heterogeneity of cancer ${ }^{15,16,31}$ can influence the cellular responses to environmental conditions. For example, a consistent increase in cell death was observed in 9 of 11 cell types for RPMI1640, whereas an increase in cell death was observed only in 3 of 11 cell types in the lower nutrient media DMEM LG, EMEM and MEM (Extended Data Fig. 4). In some conditions, cell death occurred particularly in the spheroid center, suggesting necrotic core formation (Extended Data Fig. 5).

Cellular ATP content, as a measure of metabolic activity, was lowest in the nutrient-poor medium EMEM in 8 of 11 cell types (Extended Data Fig. 6). Although commonly related to nutrient deprivation, the cause of necrotic core formation in the multicellular tumor spheroids is still controversial ${ }^{32}$. Nutrient-poor conditions correlated with increased cell death and low ATP content in some cell types (HCT116 and 4T1) but not in all. A549 spheroids cultured in the nutrient-poor media EMEM and MEM had low ATP content but decreased cell death. We measured glucose consumption and lactate secretion in supernatants of spheroids from five cell lines. This $\mathrm{L} / \mathrm{G}$ ratio was profoundly influenced by medium type in all cell types, with the lowest ratio in the high glucose-containing media DMEM/F12 and DMEMHG (Extended Data Fig. 7). In contrast, two independent studies using 2D cultured cell lines (including A549) did not observe changes in the conversion of glucose to lactate when cultured in different media ${ }^{13,16}$, suggesting the importance of 3D culture-induced nutrient gradients in these metabolic changes.

Furthermore, culture medium strongly influenced the secretion of proteins implicated in angiogenesis and immune cell interaction (Supplementary Table 5), which are crucial for tumor micro-environment (TME) communication. For example, the angiogenic and immune interactive potential of 4T1 scored high in DMEMLG, but low in DMEM/F12. In contrast, A549 scored a high TME interactivity in DMEM/F12 but a low TME interactivity in DMEM LG (Supplementary Fig. 9). Spheroid morphology, in terms of circularity, ranged between a circularity index of 0.75 and 0.95 in all tested cell lines and differed significantly between cell types (for example, the circularity index of MCF7 was $0.79 \pm 0.08$ and that of HCT116 was $0.94 \pm 0.02$ ). Intriguingly, spheroids cultured in RPMI1640 had the lowest circularity in 7 of 11 cell types (Extended Data Fig. 5 and Supplementary Fig. 10). The metric spheroid size varied significantly with medium type in 10 of 11 cell types analyzed (Extended Data Fig. 8 and Supplementary Table 6). Except for HCT116, MCF7 and SAR121, the nutrient-poor media EMEM or MEM had the smallest spheroids in all cell types analyzed (Extended Data Fig. 5). Size correlated significantly with cellular ATP content (median Pearson's $r=0.53$, two-tailed $P<0.0001$ ).

All these medium-induced changes in spheroid metrics warranted investigations into the response to a treatment intervention. Radiotherapy is used in $>50 \%$ of patients with tumors in sites such as the brain, breast, colorectum, liver and lung ${ }^{33}$, and spheroids are 
a

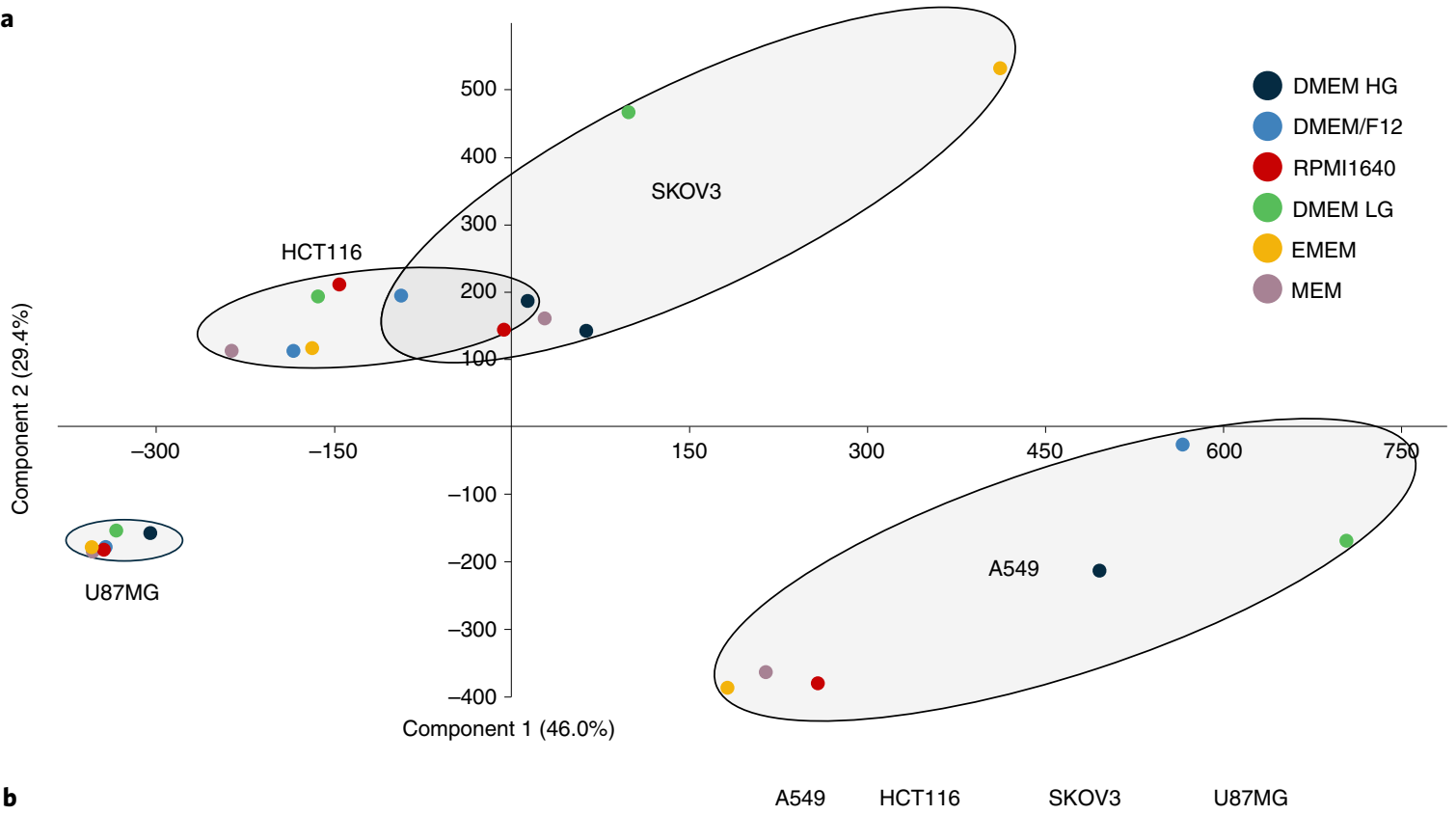

b

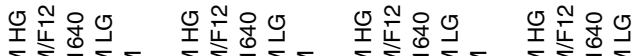

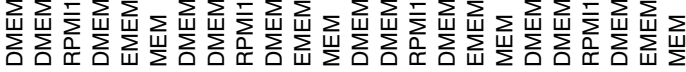

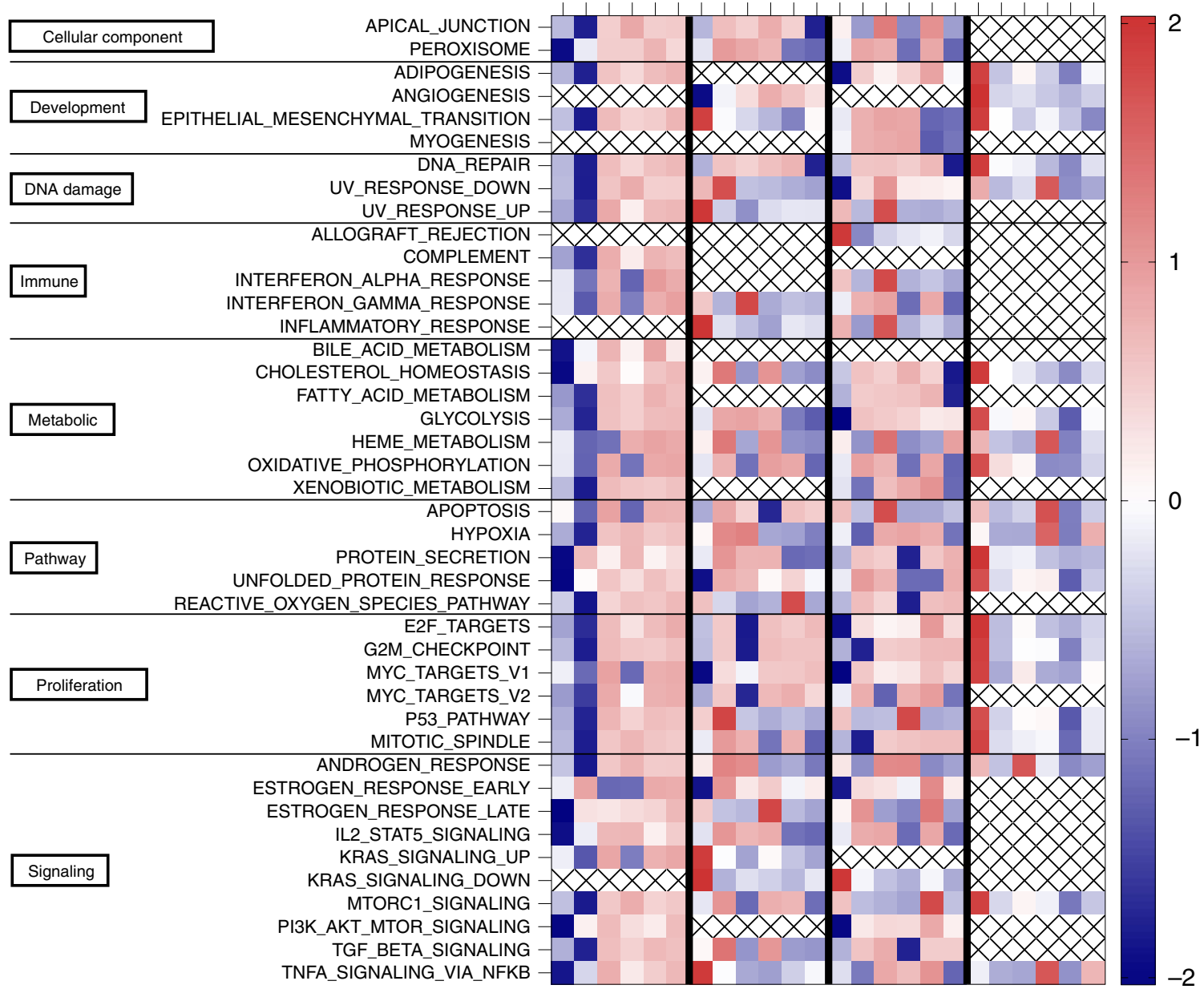

Fig. 3 | Culture media-induced transcriptional variation. a, Principal component analysis of gene expression profiles from spheroids of four cancer cell lines cultured in six different medium types. $\mathbf{b}$, Heatmap of Z-scores of all MSigDB hallmark gene sets identified by GSEA to be significantly enriched among the differentially expressed genes across the culture medium types in each cell line. Medium types are ranked from higher nutrient (left) to lower nutrient (right) richness. 
a

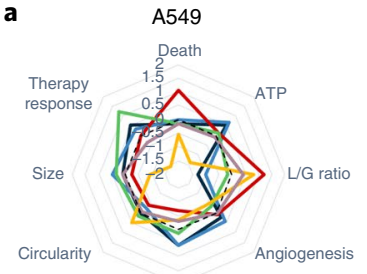

Immune
interaction

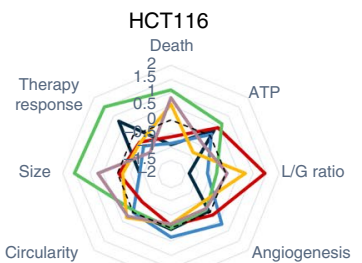

Circularity

Angiogenesis

Immune
interaction
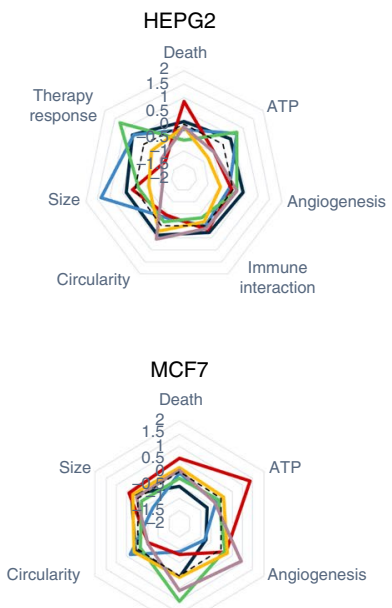

Immune

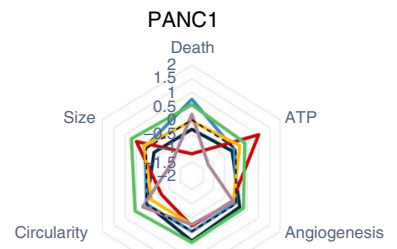

Immune

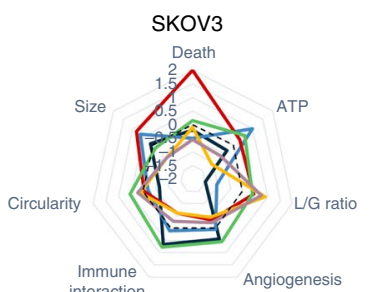

Immune
interaction
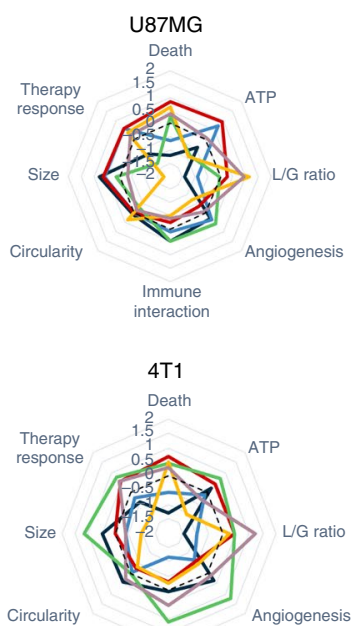

Circularity

Immune

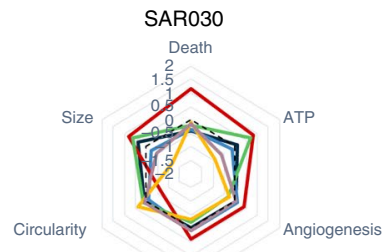

Immune

SAR120

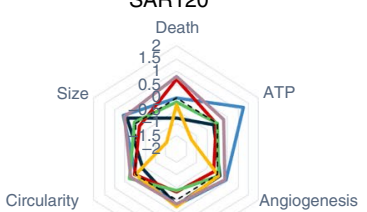

Circularity

Immune
interaction

SAR121

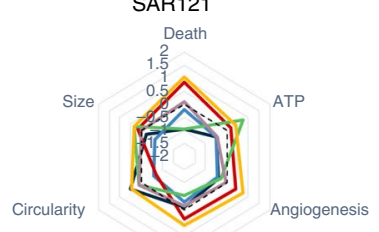

Immune

DMEM HG

DMEM/F12

RPMI1640

DMEM LG

EMEM

MEM b

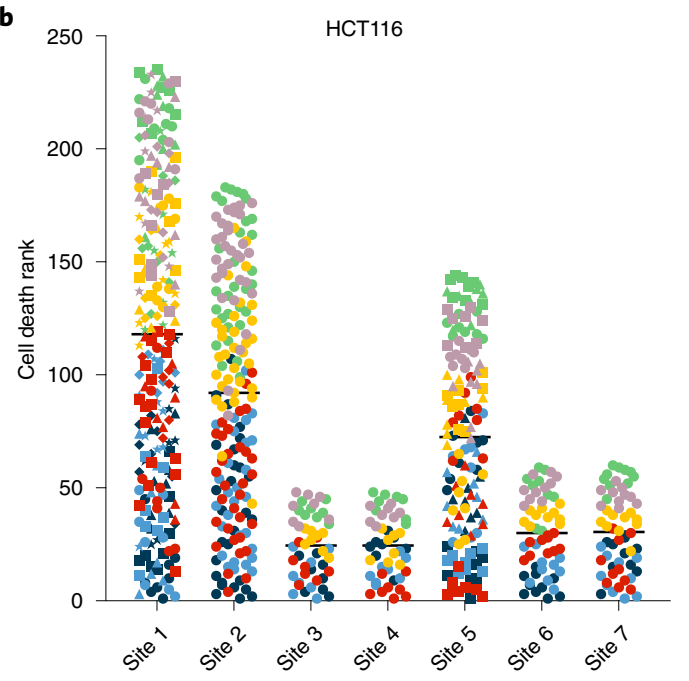

Fig. 4 | Culture media-induced heterogeneity in spheroid metrics across multiple cell types. a, Spider plots of metrics from spheroids of indicated cell lines cultured in six different medium types. Axes represent the Z-score metrics of cell death, ATP content, L/G ratio, secreted protein signatures of angiogenesis and immune interaction, circularity, size and therapy response. A higher Z-score means a higher metric value. The left and middle columns indicate established cell lines; early passage and patient-derived sarcoma cultures are on the right. b, Cell death of HCT116 spheroids cultured in six different medium types evaluated at seven different laboratories ("sites") in an interlaboratory experiment. At each site a higher ranking indicates a higher cell death. Each dot represents an evaluated spheroid. The colors indicate the medium type as in $\mathbf{a}$. 
common models to evaluate radiotherapy response ${ }^{1}$. In our evaluated cell lines, the medium type significantly influenced the radiotherapy response. Specifically, 4 of 5 investigated cell lines had the largest response to a $20 \mathrm{~Gy}$ fraction in DMEM LG culture medium (Extended Data Fig. 9a,b). The medium-dependent variability in radiotherapy response could not be explained by irreproducibility of the assay. First, biological replicate treatments yielded highly concordant results (median Pearson's $r=0.90$, two-tailed $P<0.0001$ ). Second, a $10 \mathrm{~Gy}$ fraction had similar patterns of activity across cell lines (Extended Data Fig. 9c,d).

To validate our results, we initiated an interlaboratory study to chart cell death, circularity and size in HCT116 spheroids cultured in six different media (Fig. 4b). The study used short tandem repeat (STR)-confirmed HCT116 cells, available at each participating laboratory, to allow for a realistic situation in which diversity occurred not only as a result of heterogeneity in culture media but also through differences in passage number, serum batches, pre-spheroid and laboratory-specific culture conditions, researchers and instruments. A dot plot analysis of the ranking of cell death in each analyzed spheroid (Fig. 4b) reproduced a specific pattern of media impact across different sites with a median Spearman correlation across the entire dataset of 0.91 . The median correlation between biological replicates at the study-initiating laboratory (site 1) was only slightly higher, at 0.96 (Extended Data Fig. 10a). Furthermore, in agreement with results generated in the study-initiating laboratory, RPMI1640 was validated as the medium in which spheroids had the lowest circularity (in 4 of 6 external sites), and DMEM LG was validated as the medium in which spheroids had the largest size (in 5 of 6 external sites) (Extended Data Fig. 10b,c). Consequently, the interlaboratory data on HCT116 spheroids were indicative of the robustness of the impact of medium heterogeneity on spheroid metrics and demonstrated the generalizability of our findings.

An impact of spheroid formation methods on morphology-related spheroid metrics has been shown clearly in previous reports ${ }^{28-30,34}$. The two most frequently reported formation methods (hanging drop and liquid overlay using ULA plates) in A549, HCT116, SKOV3 and 4T1 cell cultures (Supplementary Figs. 5 and 8) were compared in one medium type (DMEMLG), and this showed that ATP content, circularity and size were profoundly affected by formation methodology in a cell type-specific manner (Fig. 5a and Supplementary Fig. 11).

Spheroid size, a parameter influencing hypoxia and necrotic core formation ${ }^{1}$, is largely underreported in MISpheroID. Spheroids with different seeding cell numbers at the start of the experiment had size-dependent changes in the metrics cell death and ATP content (Fig. 5b, Supplementary Figs. 12 and 13 and Supplementary Table 6), which confirmed previous reports of the importance of spheroid size on spheroid characteristics ${ }^{10,11}$. Differences were observed in most culture media for all cell types evaluated. Intriguingly, the smallest $(<550 \mu \mathrm{m}) \mathrm{HCT} 116$ spheroids showed medium-dependent changes in ATP but not in cell death. Thus, depending on cell line, spheroid size differentially affected spheroid metrics within a certain medium type but also between medium types.

The spheroid community is challenged by heterogeneity and reporting deficiencies that cause a significant variation in the readout of one or more spheroid metrics in a cell type-specific manner. Our analyses underscore the importance of transparent reporting of experimental parameters affecting spheroid interpretation.

Creation of the MISpheroID tool. Transparency expectations to ensure experimental robustness and reproducibility have prompted the creation of reporting tools in various fields ${ }^{17,18,21-26}$. In accordance with MISpheroID knowledgebase interrogation, in-depth empirical evaluation and available supportive literature ${ }^{10-13,28-30,34}$, the MISpheroID Consortium recommends the introduction of the MISpheroID tool in the spheroid community. This tool generates a spheroid ID string consisting of four components that the Consortium argues to be the minimum information required for interpretation, comparison and replication of spheroid experiments: cell type(s) (one or multiple cell types (co-culture) included in one spheroid), culture medium (the environment in which spheroids are formed and cultivated), spheroid formation method (that is, liquid overlay (for example, ULA plates), hanging drop, spinner flask, microfluids and so on), and size (the diameter \pm s.d. of the spheroid after spheroid formation (at the moment of application)).

Typical example of an MISpheroID string. A representative example of an MISpheroID string is [4T1 - EMEM - Liquid overlay $-348 \pm 23 \mu \mathrm{m}]$.

Currently, only 300 of 1,628 (18.4\%) breast cancer-related spheroid experiments provide all four components of the MISpheroID string. Global reporting of MISpheroID string parameters shows that some are reported relatively more often (that is, cell line and spheroid formation method) than others (that is, culture medium and size). Importantly, in $80.7 \%$ of the experiments, an increase in MISpheroID string reporting can be achieved without additional experiments (correct reporting of medium type and size, based on available microscopy images).

Using the MISpheroID platform. We invite the community to upload spheroid experiments through https://www.mispheroid. org (Fig. 6). As part of each upload, 31-55 (depending on sub-questions) experimental parameters related to cell type, culture medium, spheroid formation method and size are recorded and are fully compatible with queries for deeper information. Each annotated experiment receives a MISpheroID string and unique registration code. The MISpheroID string does not reflect the quality of a spheroid experiment, nor does it impose a specific methodology, but it improves spheroid research transparency and experimental design. Users can search the knowledgebase for articles using a range of search parameters. The query result list is accompanied by a MISpheroID string and Pubmed ID (PMID). MISpheroID querying stimulates awareness and motivates researchers to compare general and specific information. We encourage researchers to provide feedback about the knowledgebase and transparency tool using the contact section of MISpheroID (Fig. 6).

\section{Discussion}

Spheroidsare attractive3D tissue structures for research purposes, and rapid progress in imaging, automated high-throughput production

Fig. 5 | Effect of formation methodology and spheroid size-induced heterogeneity on spheroid metrics across multiple cell types. a, Spider plot of metrics from spheroids of indicated cell lines generated by the hanging drop (red) or liquid overlay (green) spheroid formation method. Axes represent the Z-score metrics of cell death, ATP content, secreted protein signatures of angiogenesis and immune interaction, circularity and size. A higher Z-score means a higher metric value. $\mathbf{b}$, Violin plots representing the impact of spheroid size on cell death (upper panel) and ATP content (lower panel) metrics of HCT116 spheroids cultured in six different media. Each biological replicate has a different symbol $(N \geq 3)$, and each symbol is a technical replicate $(n=8)$. Triangles at the $X$ axis represent increasing seeding cell number and consequently increasing spheroid size; for absolute size estimates see Supplementary Table 6. The $Y$ axis represents $\log _{2}$-transformed data, and all media are normalized to DMEM HG. The horizontal bar indicates the median. Statistical significance between the groups was determined with a one-way ANOVA and Tukey's multiple comparison test. ${ }^{\star \star} P<0.01,{ }^{\star \star \star} P<0.001,{ }^{\star \star \star \star} P<0.0001$. Colors indicate medium type; media are ranked from higher nutrient (left) to lower nutrient (right) richness. 
and microfluidic technologies ensures their future implementation in drug screening, tumor biology studies and tissue engineering. However, to reach its full potential, there must be in-depth reporting of the diverse experimental parameters in the methodological setup of spheroids. This study objectively demonstrates that cell line, culture medium composition, spheroid formation method a

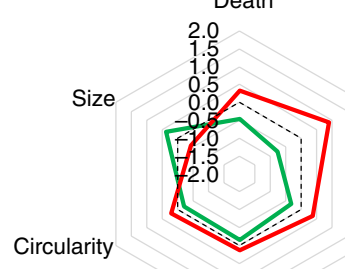

Angiogenesis

Immune

Interaction

ATP

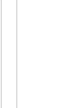

Circularity

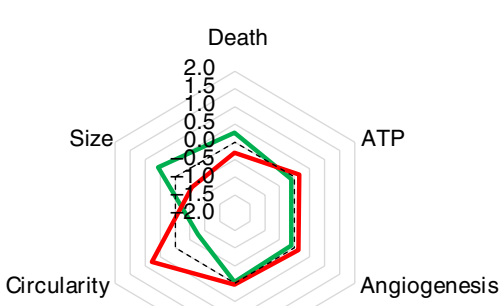

Immune

Interaction

$4 \mathrm{~T} 1$

Death

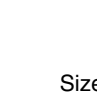

Size

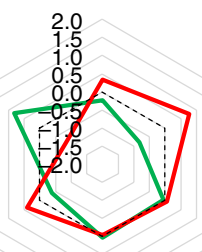

Circularity
ATP

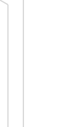

Angiogenesis
Death

Size

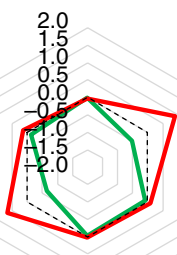

ATP

Angiogenesis
Circularity

Immune

interaction

Immune

interaction

Hanging drop

b
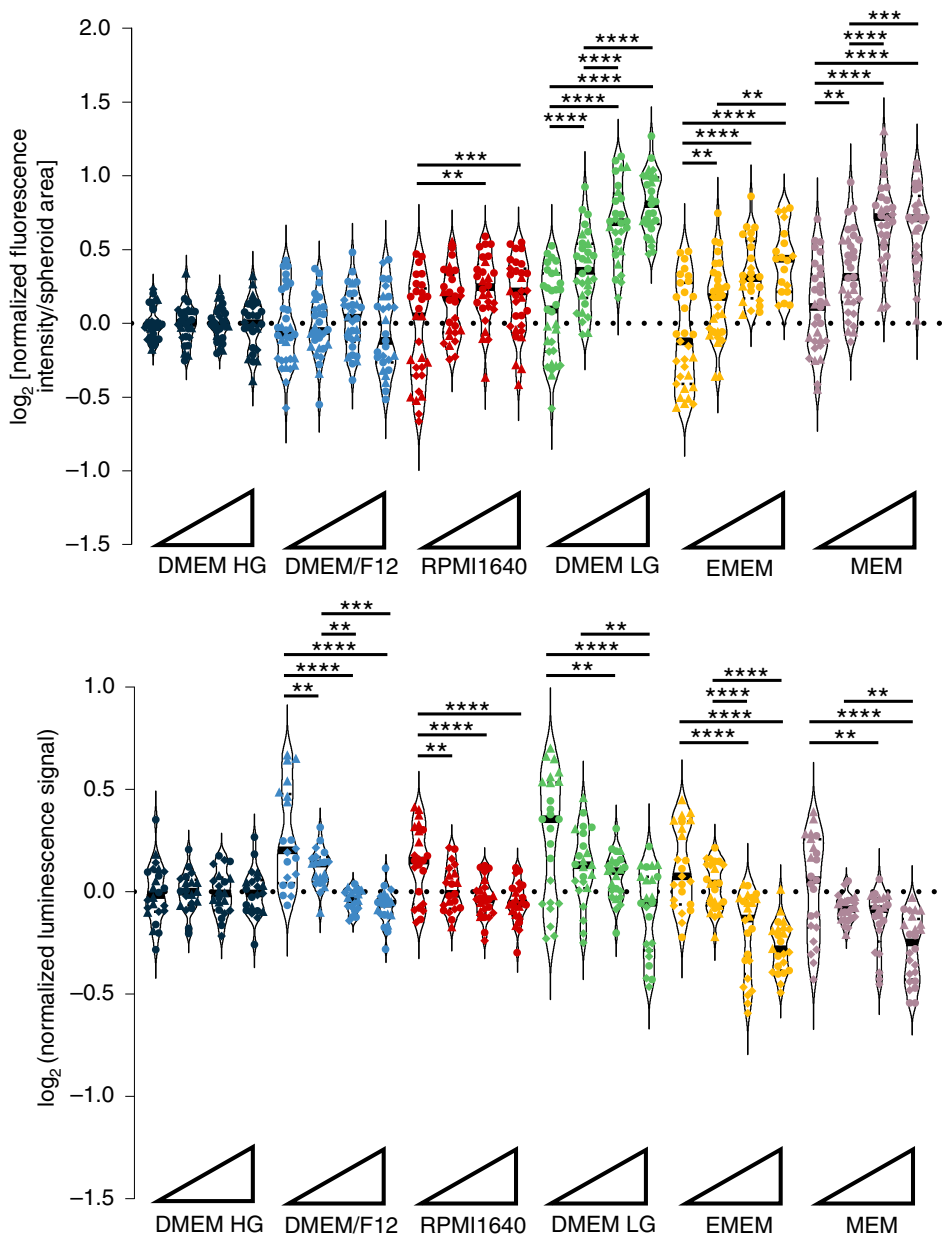


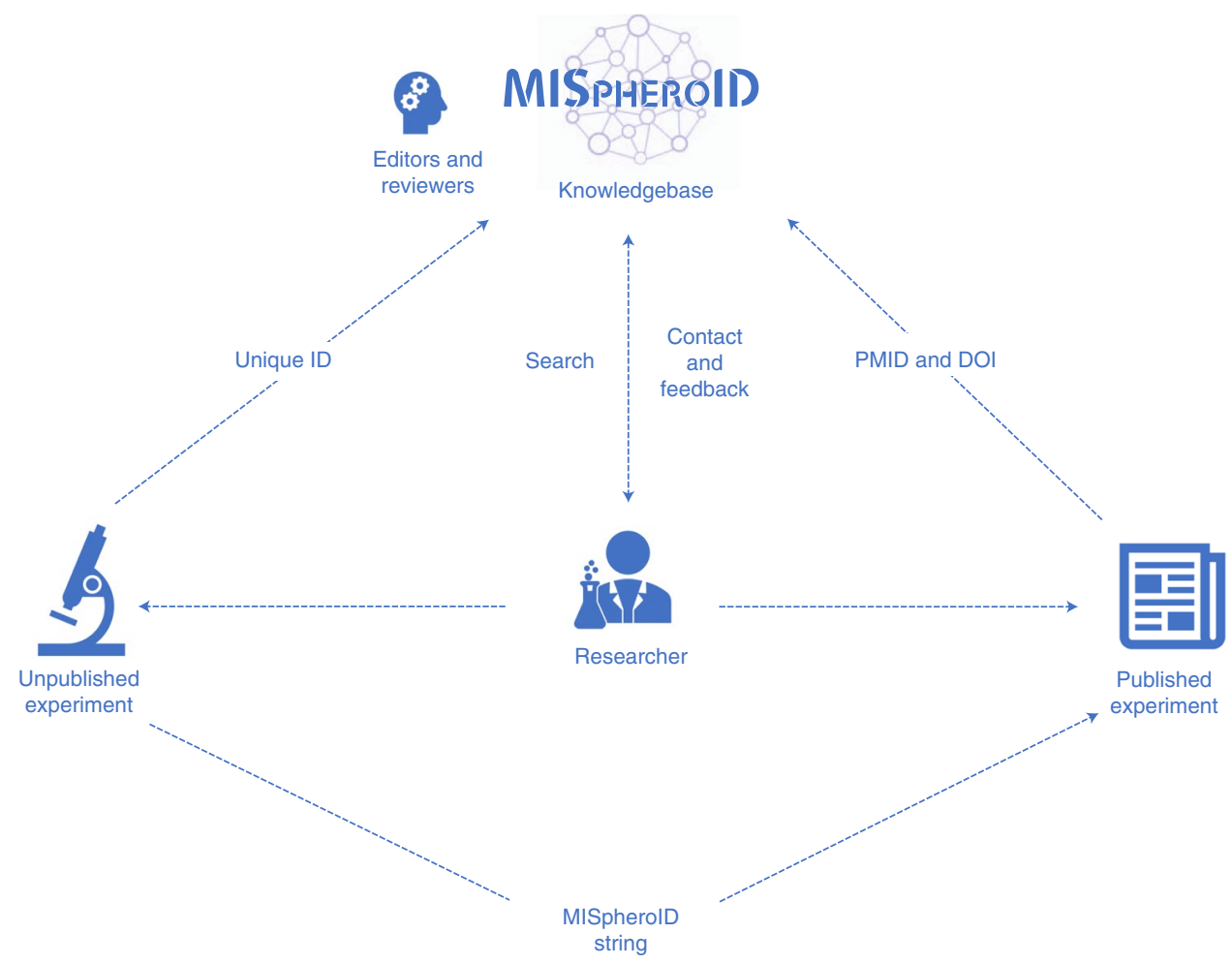

Fig. 6 | Implementation of the MISpheroID knowledgebase. This flowchart illustrates the application of MISpheroID.

and spheroid size significantly affect the phenotypic landscape of spheroids. Although it was not the Consortium's aim to identify the causes of the culture medium-induced changes in spheroid metrics, we expect that the non-physiological concentrations of some nutrients affect $3 \mathrm{D}$ biology in a cell context-dependent manner. The choice of medium type should be considered when designing studies that aim to explore pathophysiology or that aim to identify the dependency of cancer cells on specific signaling pathways, or when investigating the effects of therapeutic intervention (such as radiotherapy in this study). Most importantly, failure to understand heterogeneity can result in data that are difficult to interpret and reproduce.

The MISpheroID tool reflects the process of generating a spheroid to allow evaluation of experimental consistency and straightforward resource exchange for understanding spheroid data output (biology, drug screening and so on). Some of the challenges (cell line and culture medium) are not unique to the spheroid field and have been discussed previously ${ }^{21-23}$. We recognize that achieving a meaningful improvement in the transparency of reporting will require engagement and acceptance from all stakeholders, including investigators, reviewers, funding agencies and journal editors ${ }^{17,18,25}$.

The MISpheroID resource has its limitations, which should be considered. First, empirical data are obtained from monoculture spheroids; co-cultures were not analyzed in this study. Second, spheroid metrics were evaluated at a fixed predefined time point; longitudinal analyses were not included in the study. Third, the knowledgebase covers a high number of spheroid experiments representing cancer biology but does not cover spheroid experiments from other fields, thereby potentially underestimating the full spheroid landscape. Despite these limitations, the knowledgebase contains information on non-cancer cells present in spheroid co-culture experiments such as mesenchymal and immune cells. Considering differences in metabolic demands for non-cancer cells, the components of the MISpheroID knowledgebase and tool are equally useful for non-cancer cells. Other emerging 3D model systems, such as organoids and self-renewing multicellular aggregates that self-organize into lumen-containing ex-vivo organs, may also benefit from the MISpheroID tool to improve transparency and document heterogeneity ${ }^{35}$.

We developed MISpheroID to capture and disseminate data related to spheroid models and we will continue to evolve MISpheroID to reflect the state of the art in the field. Given the recent success of immune checkpoint inhibitors in the treatment of cancer, improving the immune component of spheroid models can be steered with knowledge accumulated in this study such as protein secretion in spheroid supernatants (for specific secretion profiles see the source data files). Recent advancements in technology will facilitate more in-depth characterization and reporting of spheroid metrics. For example, breakthroughs in submillimeter particle characterization in geology, engineering and the food industry may lead to better $3 \mathrm{D}$ characterization of spheroid sphericity and compaction ${ }^{36}$.

As has been demonstrated across multiple domains, adoption of minimum information by a research community accelerates the rate of transparency and drives scientific progress. Reporting of the minimum information required is already compulsory or recommended for publishing in several journals ${ }^{24}$. MISpheroID contributes to the field of 3D spheroid biology by providing first, a knowledgebase that catalogs spheroid setup, characterization and applications to enable the community to share and access key technical and biological insights in 3D experimentation; second, minimum information parameters combined with a tool to implement them; and last, a resource containing experimental data demonstrating the impact of experimental variations on spheroid metrics. In conclusion, the MISpheroID Consortium aims to advance 3D biology in both academic and industrial environments by removing the barriers of inconsistency while promoting reproducibility.

\section{Online content}

Any methods, additional references, Nature Research reporting summaries, source data, extended data, supplementary 
information, acknowledgements, peer review information; details of author contributions and competing interests; and statements of data and code availability are available at https://doi.org/10.1038/ s41592-021-01291-4.

Received: 7 June 2020; Accepted: 9 September 2021;

Published online: 1 November 2021

\section{References}

1. Hirschhaeuser, F. et al. Multicellular tumor spheroids: an underestimated tool is catching up again. J. Biotechnol. 148, 3-15 (2010).

2. Riedl, A. et al. Comparison of cancer cells in $2 \mathrm{D}$ vs $3 \mathrm{D}$ culture reveals differences in AKT-mTOR-S6K signaling and drug responses. J. Cell Sci. 130, 203-218 (2017).

3. Laschke, M. W. \& Menger, M. D. Life is 3D: boosting spheroid function for tissue engineering. Trends Biotechnol. 35, 133-144 (2017).

4. Madoux, F. et al. A 1536-well 3D viability assay to assess the cytotoxic effect of drugs on spheroids. SLAS Discov. 22, 516-524 (2017).

5. Selby, M. et al. 3D models of the NCI60 cell lines for screening oncology compounds. SLAS Discov. 22, 473-483 (2017).

6. Kota, S. et al. A novel 3-dimensional high throughput screening approach identifies inducers of a mutant KRAS selective lethal phenotype. Oncogene 37, 4372-4384 (2018)

7. Carragher, N. et al. Concerns, challenges and promises of high-content analysis of 3D cellular models. Nat. Rev. Drug Discov. 17, 606 (2018).

8. Han, K. et al. CRISPR screens in cancer spheroids identify 3D growth-specific vulnerabilities. Nature 580, 136-141 (2020).

9. Weiswald, L. B., Bellet, D. \& Dangles-Marie, V. Spherical cancer models in tumor biology. Neoplasia 17, 1-15 (2015).

10. Zanoni, M. et al. 3D tumor spheroid models for in vitro therapeutic screening: a systematic approach to enhance the biological relevance of data obtained. Sci. Rep. 6, 1-11 (2016).

11. Däster, S. et al. Induction of hypoxia and necrosis in multicellular tumor spheroids is associated with resistance to chemotherapy treatment. Oncotarget 8, 1725-1736 (2017)

12. Wenzel, C. et al. 3D high-content screening for the identification of compounds that target cells in dormant tumor spheroid regions. Exp. Cell Res. 323, 131-143 (2014)

13. Vande Voorde, J. et al. Improving the metabolic fidelity of cancer models with a physiological cell culture medium. Sci. Adv. 5, 7314 (2019).

14. Dontu, G. et al. In vitro propagation and transcriptional profiling of human mammary stem/progenitor cells. Genes Dev. 17, 1253-1270 (2003).

15. Cantor, J. R. \& Sabatini, D. M. Cancer cell metabolism: one hallmark, many faces. Cancer Discov. 2, 881-898 (2012).

16. Cantor, J. R. et al. Physiologic medium rewires cellular metabolism and reveals uric acid as an endogenous inhibitor of UMP synthase. Cell $\mathbf{1 6 9}$ 258-272 (2017)

17. Begley, C. G. \& Ellis, L. M. Raise standards for preclinical cancer research. Nature 483, 531-533 (2012)

18. Landis, S. C. et al. A call for transparent reporting to optimize the predictive value of preclinical research. Nature 490, 187-191 (2012).

19. Moscona, A. \& Moscona, H. The dissociation and aggregation of cells from organ rudiments of the early chick embryo. J. Anat. 86, 287-301 (1952).

20. Sutherland, R., McCredie, J. \& Inch, W. Growth of multicell spheroids in tissue culture as a model of nodular carcinomas. J. Natl Cancer Inst. 46, 113-120 (1971).
21. Taylor, C. F. et al. Promoting coherent minimum reporting guidelines for biological and biomedical investigations: the MIBBI project. Nat. Biotechnol. 26, 889-896 (2008).

22. FAIRsharing.org MIACA: minimal information about a cellular assay. https:// doi.org/10.25504/FAIRsharing.7d0yv9 (2019).

23. FAIRsharing.org MIACME: minimum information about cell migration experiment. https://doi.org/10.25504/FAIRsharing. vh2ye1 (2019).

24. Théry, C. et al. Minimal information for studies of extracellular vesicles 2018 (MISEV2018): a position statement of the International Society for Extracellular Vesicles and update of the MISEV2014 guidelines. J. Extracell. Vesicles 7, 1535750 (2018).

25. Van Deun, J. et al. EV-TRACK: transparent reporting and centralizing knowledge in extracellular vesicle research. Nat. Methods $\mathbf{1 4}$ 228-232 (2017).

26. Meehan, T. F. et al. PDX-MI: minimal information for patient-derived tumor xenograft models. Cancer Res. 77, e62-e66 (2017).

27. Liberzon, A. et al. The Molecular Signatures Database (MSigDB) hallmark gene set collection. Cell Syst. 1, 417-425 (2015).

28. Gencoglu, M. F. et al. Comparative study of multicellular tumor spheroid formation methods and implications for drug screening. ACS Biomater. Sci. Eng. 4, 410-420 (2018).

29. Amaral, R. L. F., Miranda, M., Marcato, P.D. \& Swiech, K. Comparative analysis of 3D bladder tumor spheroids obtained by forced floating and hanging drop methods for drug screening. Front. Physiol. 8, 605 (2017).

30. Raghavan, S. et al. Comparative analysis of tumor spheroid generation techniques for differential in vitro drug toxicity. Oncotarget 7 , 16948-16961 (2016).

31. Eason, K. \& Sadanandam, A. Molecular or metabolic reprograming: what triggers tumor subtypes? Cancer Res. 76, 5195-5200 (2016).

32. Bertuzzi, A., Fasano, A., Gandolfi, A. \& Sinisgalli, C. Necrotic core in EMT6/ Ro tumour spheroids: is it caused by an ATP deficit? J. Theor. Biol. 262, 142-150 (2010).

33. De Ruysscher, D. et al. Radiotherapy toxicity. Nat. Rev. Dis. Primers $\mathbf{5}$, 13 (2019).

34. Bresciani, G. et al. Evaluation of spheroid 3D culture methods to study a pancreatic neuroendocrine neoplasm cell line. Front. Endocrinol. 10, 682 (2019).

35. Bock, C. et al. The organoid cell atlas. Nat. Biotechnol. 39, 13-17 (2021).

36. Bagheri, G. H., Bonadonna, C., Manzella, I. \& Vonlanthen, P. On the characterization of size and shape of irregular particles. Powder Technol. 270, 141-153 (2015)

Publisher's note Springer Nature remains neutral with regard to jurisdictional claims in published maps and institutional affiliations.

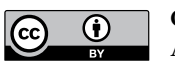

Open Access This article is licensed under a Creative Commons Attribution 4.0 International License, which permits use, sharing, adaptation, distribution and reproduction in any medium or format, as long as you give appropriate credit to the original author(s) and the source, provide a link to the Creative Commons license, and indicate if changes were made. The images or other third party material in this article are included in the article's Creative Commons license, unless indicated otherwise in a credit line to the material. If material is not included in the article's Creative Commons license and your intended use is not permitted by statutory regulation or exceeds the permitted use, you will need to obtain permission directly from the copyright holder. To view a copy of this license, visit http://creativecommons. org/licenses/by/4.0\%

() The Author(s) 2021 


\section{Methods}

Literature search. Medline (PubMed) was searched for articles applying active spheroid formation methods with cancer cells (cell lines and early passage patient-derived cell cultures). Therefore, the following key terms were applied: spheroid (and derived terms), cancer, neoplasm, tumor and 'organ' (brain, breast, colorectum, liver, lung, ovary and pancreas). We focused on active spheroid formation, therefore at abstract check, reviews and letters were excluded as well as articles that did not involve active spheroid formation or those in which spheroids did not include organ-specific cells. At full-text screening, we applied the same exclusion criteria and excluded articles for which the full text was not available. Spheroids from multiple cell lines or spheroid formation methods were considered as a separate experiment. For the breast cancer spheroid-related articles used to set up the knowledgebase, published articles from 1979 to 2020 were evaluated. For the spheroid-related articles from other organ tumor types, published articles from 2018 to 2021 were evaluated. As an example, a detailed flow chart for literature screening of breast of breast cancer spheroids is provided in Supplementary Fig. 1.

Experimental setup. An overview schematic (Supplementary Fig. 14) explains the use of different cell types, methodological setups and metrics evaluated in the study as detailed in the Methods section.

Cell culture. The cell lines A549 (cat. no. CCL-185), HCT116 (cat no. CCL-247), HEPG2 (cat. no. HB-8065), MCF7 (cat. no. HTB-22), PANC1 (cat. no. CRL-1469), SKOV3 (cat. no. HTB-77), U87MG (cat. no. HTB-14) and 4T1 (cat. no. CRL-2539) were purchased from the American Type Culture Collection. To establish the early passage cell cultures (SAR030, SAR120 and SAR121), patient-derived sarcoma samples (Supplementary Table 7 ) were cut into pieces measuring $1-2 \mathrm{~mm}^{3}$. The pieces were digested in $500 \mathrm{U} \mathrm{ml}^{-1}$ collagenase II solution (cat. no. 17101015, ThermoFisher) and $22 \mathrm{KU} \mathrm{ml}^{-1}$ DNase I solution (cat. no. A3778.0010, VWR) and processed according to the Gentlemax tumor digestion protocol (Miltenyi Biotec). The cell suspension was applied to a cell strainer $(100 \mu \mathrm{m}$, cat. no. 352360, Corning), centrifuged at $300 \times \mathrm{g}$ for $5 \mathrm{~min}$ and, after aspiration of the supernatant and removal of remnant red blood cells, maintained in culture. All human cell lines were authenticated using a 21-Marker STR Profile test (Eurofins) and tested monthly using the Mycoalert Mycoplasma Detection Kit (cat. no. LT07-318, Lonza) to exclude mycoplasma contamination. All cells were cultured in DMEM (cat. no. 41965039, ThermoFisher) supplemented with $10 \%$ heat-inactivated fetal bovine serum (FBS) (cat. no. ATCC-30-2030, LGC Standards), $100 \mathrm{IU} \mathrm{ml}^{-1}$ penicillin and $100 \mathrm{mg} \mathrm{ml}^{-1}$ streptomycin (cat. no. 15070063 , ThermoFisher). Cells were expanded and maintained as a monolayer at $37^{\circ} \mathrm{C}$ in an atmosphere of $5 \% \mathrm{CO}_{2}$ in air and passaged at $80 \%$ confluence.

Spheroid formation. The U-shaped, 384-well ULA plates (cat. no. MS-9384UZ, S-bio) were seeded with a suspension of $80 \mu$ l cell culture media with $2 \times 10^{3}$ cells per well (A549, HCT116, HEPG2, MCF7, PANC1, SKOV3, U87MG, 4T1, SAR120, SAR121) or $8 \times 10^{3}$ cells per well (SAR030) in $2 \times 8$ technical replicates per condition. The culture media used were DMEM HG $\left(4.5 \mathrm{gl}^{-1}=25 \mathrm{mM}\right.$ glucose $)$ (cat. no. 41965039, ThermoFisher), DMEM/F12 (1:1) $\left(3.15 \mathrm{gl}^{-1}=17.5 \mathrm{mM}\right.$ glucose) (cat. nos. 41965039 and 21765029, ThermoFisher), RPMI1640 $\left(2 \mathrm{gl}^{-1}=11.1 \mathrm{mM}\right.$ glucose) (cat. no. 21875091, ThermoFisher), DMEMLG $\left(1 \mathrm{gl}^{-1}=5.6 \mathrm{mM}\right.$ glucose) (cat. no. 31885023 , ThermoFisher), EMEM $\left(1 \mathrm{gl}^{-1}=5.6 \mathrm{mM}\right.$ glucose) (cat. no. 10-009-CV, Corning) and MEM $\left(1 \mathrm{gl}^{-1}=5.6 \mathrm{mM}\right.$ glucose) (cat. no. $10370-047$, ThermoFisher), all supplemented with $10 \% \mathrm{FBS}, 100 \mathrm{IU} \mathrm{ml}^{-1}$ penicillin and $100 \mathrm{mg} \mathrm{ml}^{-1}$ streptomycin. Note that $5.6 \mathrm{mM}$ glucose is the physiological plasma glucose concentration. MEM was supplemented with $2 \mathrm{mM}$ L-glutamine. Extended Data Fig. 3 provides extensive details on the nutrient content of 10 culture media reported in MISpheroID, of which six were empirically evaluated. The 384-well ULA plates were sealed with Breathe-Easy semipermeable tape (cat. no. BEM-1, Diversified Biotech) to prevent evaporation. The spheroids were cultured at $37^{\circ} \mathrm{C}$ in an atmosphere of $5 \% \mathrm{CO}_{2}$ under normoxia.

For the comparison of spheroid size, $2 \times 10^{3}, 4 \times 10^{3}, 6 \times 10^{3}$ or $8 \times 10^{3}$ cells (A549 and SKOV3) and $0.5 \times 10^{3}, 1 \times 10^{3}, 2 \times 10^{3}$ or $3 \times 10^{3}$ cells (HCT116) were seeded in $80 \mu \mathrm{l}$ cell culture media in the U-shaped, 384-well ULA plates. The culture media used were DMEM HG, DMEM/F12, RPMI1640, DMEM LG, EMEM and MEM.

For the comparison of the liquid overlay method and the hanging drop method, $2 \times 10^{3}$ cells (A549, HCT116, SKOV3, 4T1) were seeded in $80 \mu \mathrm{l}$ cell DMEMLG in U-shaped 96-well ULA plates (cat. no. CLS7007-24EA, Sigma-Aldrich) in the liquid overlay method. In the hanging drop method, $2 \times 10^{3}$ cells were plated under the lids of petri dishes (cat. no. A19618, Novolab) in $20 \mu \mathrm{l}$ drops of DMEM LG (50 technical replicates). The bottom of the petri dishes was filled with $10 \mathrm{ml}$ PBS (phosphate buffered saline; cat. no. 20012019, ThermoFisher) to limit evaporation.

RNA sequencing. Spheroids from four independent cell lines using four technical replicates from one biological replicate were used and cultured in 384-well ULA plates in six different cell culture media (a total of 96 conditions) for $5 \mathrm{~d}$ (HCT116) or $7 \mathrm{~d}$ (A549, SKOV3 and U87MG). RNA extraction was performed on two spheroids per condition using the miRNeasy Micro Kit (cat. no. 217084
Qiagen). RNA-seq libraries were prepared from purified RNA using the QuantSeq 3' mRNA-Seq Library Prep Kit FWD for Illumina (Lexogen) according to the manufacturer's instructions, with $27.5 \mathrm{ng}$ RNA that was treated with Heat-Labile Double Strand-specific DNase (Arcticzymes). The individual libraries were quantified by qPCR using the KAPA Library Quantification Kit (Roche) and equimolarly pooled. The pool concentration was measured with Qubit, and sequencing was carried out at a concentration of $1.4 \mathrm{pM}$ with $1 \% \mathrm{PhiX}$ on a NextSeq 500 (Illumina) (NextSeq software v.4.0.1) using a high-output $1 \times 75$ run. Reads were mapped to the human genome using Tophat and gene expression counts were generated using HTSeq.

Data normalization and gene set enrichment analysis. Normalization and differential gene expression analysis were performed using DESeq2 (v1.30.1). Terms from the Molecular Signatures Database (MSigDB) Hallmark Gene Signatures ${ }^{27}$ were used for enrichment analysis with GSEA software (v4.1.0). We tested gene sets for significant enrichment among the differentially expressed genes between the six medium types used to culture the spheroids in each cell line, to assess how they might differ from each other in terms of molecular pathways. Statistical significance between the different media for all hallmarks was determined for every cell line using one-way analysis of variance (ANOVA) and Tukey's multiple comparison test with the alpha level of significance set at 0.05 . Principal component analysis with the Manhattan distance index was performed using PAST4.03.

Cell death staining. Spheroids were cultured in the 384-well ULA plates in the different cell culture media for $5 \mathrm{~d}$ (HCT116) or $7 \mathrm{~d}$ (all other cell lines). For the comparison of the liquid overlay method and the hanging drop method, spheroids were cultured for $3 \mathrm{~d}$. A total of $60 \mu \mathrm{l}$ medium per well was replaced with $10 \mu \mathrm{M}$ Ethidium homodimer I solution (cat. no. 300519, Santa Cruz) in PBS supplemented with $\mathrm{Ca}^{2+}$ and $\mathrm{Mg}^{2+}$. The hanging drop spheroids were transferred to the 96-well ULA plate and $60 \mu \mathrm{l} 10 \mu \mathrm{M}$ Ethidium homodimer I solution was added. The fluorescence signal was observed after $15 \mathrm{~min}$ incubation time at $20^{\circ} \mathrm{C}$ room temperature with an Axiovert $200 \mathrm{M}$ fluorescence microscope (Carl Zeiss) using AxioVision release 4.8. The average dead signal was measured over the spheroid area in ImageJ (v1.52v.). Analysis was performed on a minimum of three biological replicates, with each biological replicate having eight technical replicates per condition. Statistical significance between the groups was determined with a one-way ANOVA and Tukey's multiple comparison test with the alpha level of significance set at 0.05 .

Interlaboratory study. The study-initiating laboratory sent culture media, 384-well ULA plates and Breathe-Easy semipermeable tape to six different laboratories that were part of the international MISpheroID Consortium. To perform the experiments, the laboratories added their own batches of serum to the culture media. All cells were authenticated by STR profiling. To conduct the dead staining, laboratories applied in-house optimized dead staining protocols, after which the raw data concerning dead signal, spheroid circularity and size were analyzed by the study-initiating laboratory.

ATP assay with CellTiter-Glo 3D. Spheroids were cultured in 384-well ULA plates in the different cell culture media for $5 \mathrm{~d}$ (HCT116) or $7 \mathrm{~d}$ (all other cell lines). For the comparison of the liquid overlay method and the hanging drop method, spheroids were cultured for $3 \mathrm{~d}$. Individual spheroids in the culture medium were pipetted into white micro 96-well plates (cat. no. 236108, ThermoFisher) and an equal volume of CellTiter-Glo 3D (cat. no. G9683, Promega) reagent was added. The contents were mixed for $5 \mathrm{~min}$ on an orbital shaker to induce cell lysis, while shielded from light. Luminescence readout (Gen5 Data Analysis Software v3.08.01) was performed after $25 \mathrm{~min}$ incubation at $20^{\circ} \mathrm{C}$ (room temperature). Analysis was performed on a minimum of three biological replicates, with each biological replicate having eight technical replicates per condition. Statistical significance between the groups was determined with a one-way ANOVA and Tukey's multiple comparison test with significance level alpha 0.05 .

Irradiation. Spheroids were cultured in the 384-well ULA plates in the different cell culture media for $3 \mathrm{~d}$ before being irradiated with 10 or $20 \mathrm{~Gy}$ single fractions with $6 \mathrm{MV}$ photons from an Elekta Synergy linear accelerator (Elekta). Irradiation response was measured and calculated after $2 \mathrm{~d}$ (HCT116) or $4 \mathrm{~d}$ (SKOV3, U87MG and 4T1) with the percentage change in metabolic activity (CellTiter-Glo3D) compared with non-irradiated control spheroids. Analysis was performed on a minimum of eight technical replicates per condition. Statistical significance between the groups was determined with a one-way ANOVA and Tukey's multiple comparison test with the alpha level of significance set at 0.05 .

Measurement of glucose and lactate. Endpoint concentrations of glucose and lactate in the spheroid supernatants were measured using enzymatic assays involving bioluminescent $\mathrm{NADH}$ detection technology and a selective dehydrogenase (Glucose-Glo J6021 and Lactate-Glo J5021 assay; Promega). For the glucose-Glo assay, supernatants of spheroids cultured in DMEM HG and DMEM/F12 were diluted 1:500 in PBS, and supernatants of spheroids cultured 
in RPMI1640, DMEMLG, EMEM and MEM were diluted 1:200 in PBS. For the lactate-Glo assay, supernatants of spheroids were diluted 1:100 in PBS. Controls (medium without cells) were diluted correspondingly. A $50 \mu \mathrm{l}$ sample was pipetted into white micro 96-well plates (cat. no. 236108, ThermoFisher) and an equal volume of assay reagent was added. The contents were mixed for $30 \mathrm{~s}$ on an orbital shaker while shielded from light. Luminescence readout (Gen5 Data Analysis Software v3.08.01) was performed after $1 \mathrm{~h}$ incubation at room temperature. Glucose consumption and lactate production were calculated by subtraction of the glucose or lactate concentrations of the medium without cells. Analysis was performed on a minimum of two biological replicates with each biological replicate having four technical replicates per condition. Statistical significance between the groups was determined with a Kruskal-Wallis test with Dunn's correction, with the alpha level of significance set at 0.05 .

Luminex. Spheroids were cultured in the 384-well ULA plates in the different cell culture media for $5 \mathrm{~d}$ (HCT116) or $7 \mathrm{~d}$ (all other cell lines). For the comparison of the liquid overlay method and the hanging drop method, spheroids were cultured for $3 \mathrm{~d}$. Eight technical replicates (liquid overlay method) or 42 technical replicates (hanging drop method) were then collected and the supernatant was passed through a $0.2 \mu \mathrm{m}$ filter (cat. no. A37111, Novolab) and processed with the Human Cytokine/Chemokine Array 71-Plex Panel and the Human Angiogenesis Array and Growth Factor 17-Plex Array by Eve Technologies. The supernatant collected from the 4T1 mouse mammary gland cell line was processed with the Mouse Cytokine/Chemokine Array 44-Plex Panel. Analysis was performed on a minimum of three biological replicates per condition with subtraction of the growth factor concentrations of medium without cells. Growth factors were grouped based on their GO-Biological processes related to immune interaction or angiogenesis via the UniProt Knowledgebase.

Size and circularity calculations. Spheroid size (diameter) and circularity were determined using AnaSP v1.4 (ref. $\left.{ }^{37}\right)$. The spheroid size was described by the equivalent diameter (ED). The ED is the diameter of a circle with the same projected area as the spheroid, calculated by

$$
\mathrm{ED}=2 \sqrt{ }(\operatorname{area} / \pi)
$$

The spheroid circularity was determined by the formula:

$$
\text { circularity }=4 \pi \times \text { area/ }(\text { convex perimeter })^{2}
$$

\section{(2) Author contributions}

with $x$ being the value of every replicate, $\mu$ being the average of the population (all replicates over the different media), and $\sigma$ being the standard deviation of the population (all replicates over the different media).

The Z-score of a culture medium was subsequently calculated as the average of the Z-scores for that culture medium.

Outlier exclusion. Data points were first excluded manually (for example, spheroids containing a dust particle when measuring dead signal, spheroids that were lost when pipetting to a white plate for measuring metabolic activity with CellTiter-Glo 3D and so on). Afterwards, outliers were calculated via the ROUT method $(\mathrm{Q}=1 \%)$ with Graphpad Prism v8.4.3, taking into account that the number of datapoints plotted in the graphs can be lower than $N \times n$ due to outlier exclusion.

Statistical analysis. Statistical analysis was performed in Graphpad Prism v8.4.3. Unpaired comparisons were conducted using a one-way ANOVA or KruskalWallis test (after the mentioned transformations and the Shapiro-Wilk assessment for normality) with Tukey's or Dunn's multiple comparison test. Comparisons of the spheroid formation method were conducted using unpaired Student's $t$-test
A.P., E.B. and O.D.W. designed the study and interpreted the data. E.B. and A.P. performed the experiments. T.A., F.C., J.D., A.D., S.Mi., J.R., E.S., C.W., N.Z. and M.Z. participated in the interlaboratory study. E.B., A.P., M.V.W. and F.Pi. analyzed the data. A.P., E.B., E.D.V., S.Maes, C.M. and M.V.W. annotated data. A.P., S.D., E.B. and O.D.W. developed the online tool. J.V. and P.M. designed and supervised the RNA-seq experiment, N.Y. and E.B. prepared the RNA-seq libraries and initiated the sequencing, and J.A., C.P. and S.D. performed the RNA-seq data analysis. All authors discussed, prepared and approved the final manuscript.

with Welch's correction. Correlation analysis was conducted by determination of Pearson or non-parametric Spearman correlation coefficients. The significance shown only for significance levels $\leq 0.01$.

Reporting Summary. Further information on research design is available in the Nature Research Reporting Summary linked to this article.

\section{Data availability}

RNA-seq data generated during the current study are available in the ArrayExpress Figs. 9-13 and Supplementary Table 6 are provided as a source data file. All literature study data are available through the MISpheroID knowledgebase. Source data are provided with this paper.

\section{References}

. Piccinini, F. AnaSP: a software suite for automatic image analysis of multicellular spheroids. Comput. Methods Programs Biomed. 119, 43-52 (2015).

\section{Acknowledgements}

This work was supported by the Concerted Research Actions from Ghent University Kanker (Stand up to Ctichting Tegen Kanker (grant no. 2020-073), Kom Op Teg Research Flanders (grant no. 1198121N) (O.D.W.); EU Horizon2020 project LSFM4LIFE (grant no. 668350-2), the EU FET-OPEN project BRIGHTER (grant no. 828931) and the ZonMw-BMBF joint sponsored project Onconoid Hub (grant no 114027003) (F.Pa.); (grant no. GN-000676) (N.C.); US National Institutes of Health (NIH) (grant no. R01 CA196018 and R21 AG061687) (S.T.); Sigrid Juséliuksen Foundation and Cancer Society of Finland (J.H.); Canadian Institutes of Health Research (CIHR) (grant no. 147081) (S.M.R.); German Federal Ministry of Education and Research ((BMBF), grant no. 13N11115 (ProMEBS)) (P.S.); and German Federal Ministry of Education and Research

\section{Competing interests}

The authors declare no competing interests.

Extended data are available for this paper at https://doi.org/10.1038/ s41592-021-01291-4.

Supplementary information The online version contains supplementary material available at https://doi.org/10.1038/s41592-021-01291-4.

Correspondence and requests for materials should be addressed to Olivier De Wever Peer review information Nature Methods thanks Tim Downing and the other, anonymous, reviewer(s) for their contribution to the peer review of this work. Madhura Mukhopadhyay was the primary editor on this article and managed its editorial process and peer review in collaboration with the rest of the editorial team.

Reprints and permissions information is available at www.nature.com/reprints. (www.ebi.ac.uk/arrayexpress) under accession number E-MTAB-10862.

\section{Additional information}




\section{MCF7}

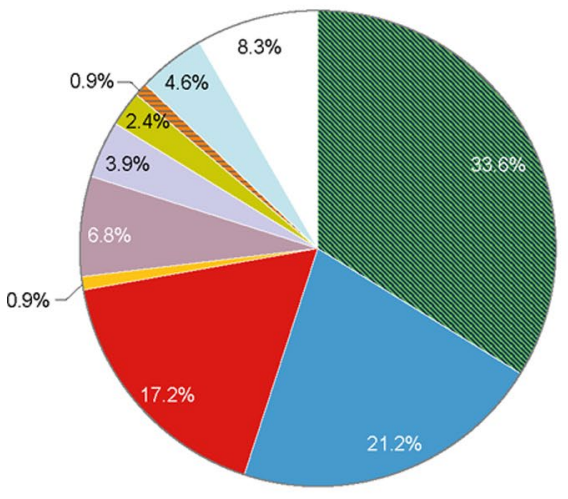

$N=542$

T47D

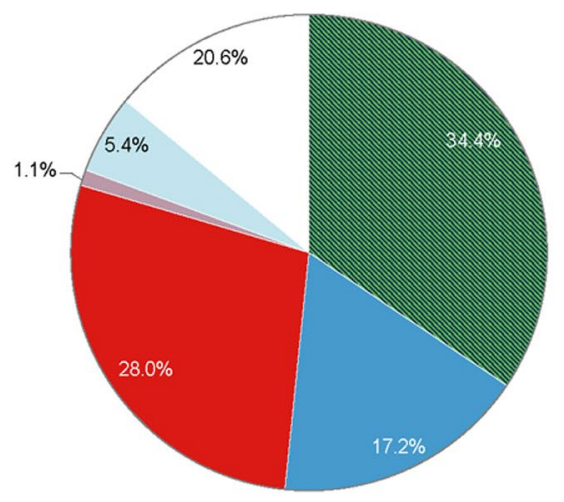

$\mathrm{N}=93$

\section{MDAMB231}

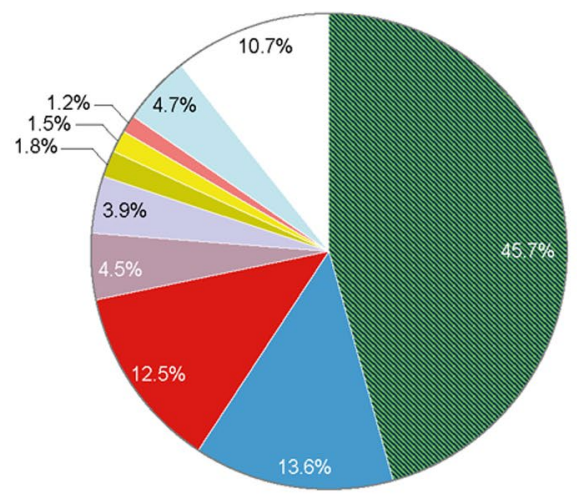

$\mathrm{N}=337$

4T1

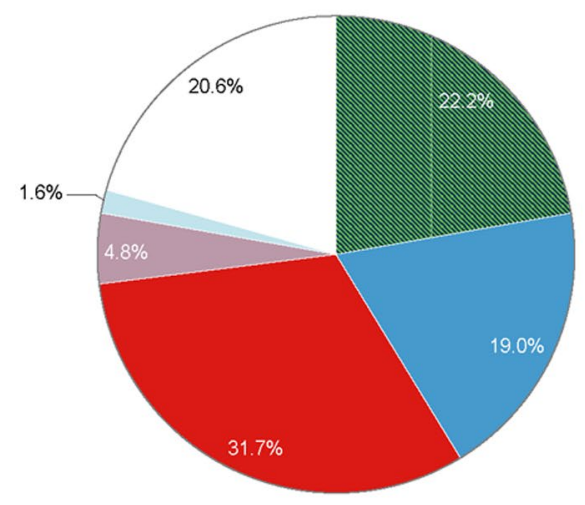

$\mathrm{N}=63$

DMEM $\square$ DMEM/F12 $\square$ RPMI1640 $\square$ EMEM $\square$ MEM
Mammocult $\Omega \square$ MEGM $\square$ MEBM $\square$ IMDM $\square$ L15 $\square$ Other $\square$ Not Specified

Extended Data Fig. 1 | Distribution of the applied culture medium to prepare spheroids of the estrogen-dependent MCF7 and T47D, and triple-negative MDAMB231 and 4T1 breast/mammary gland cancer cell lines. Pie chart visualizing the proportion of culture media used to prepare MCF7, T47D, MDAMB231 and 4T1 spheroids. 
A549

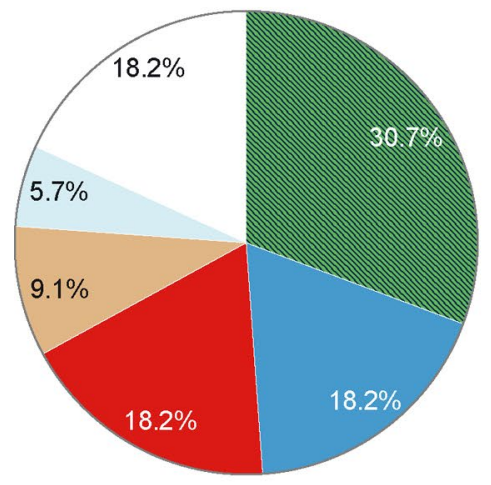

$N=88$

HEPG2

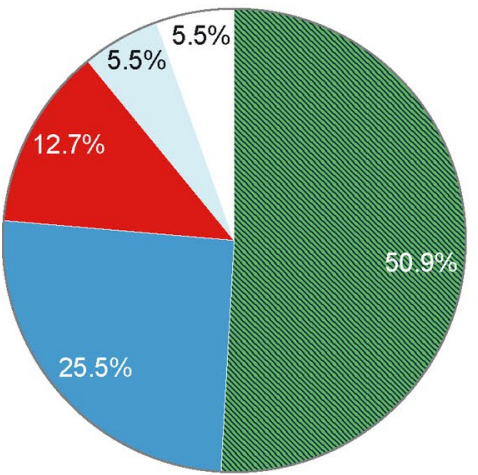

$N=55$

SKOV3

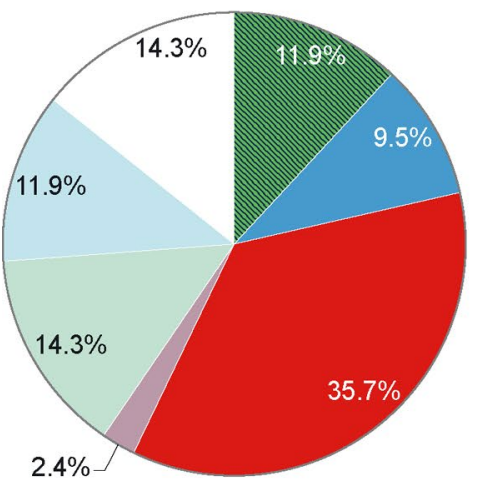

$\mathrm{N}=42$
HCT116

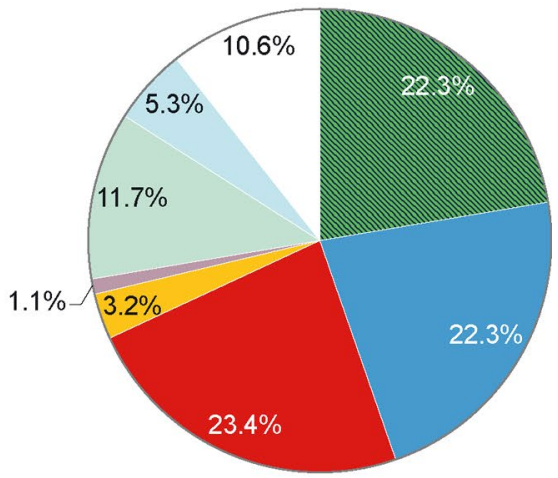

$\mathrm{N}=94$

PANC1

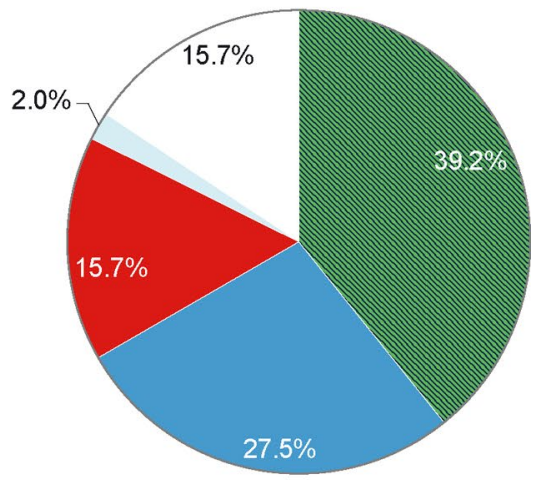

$\mathrm{N}=51$

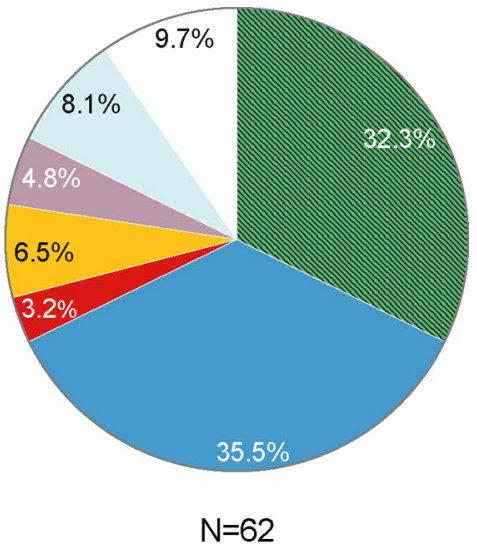

DMEM $\square$ DMEM/F12 $\square$ RPMI1640 $\square$ EMEM $\square$ MEM
$\square$ HamF12K $\square$ McCoy's5A $\square$ Other $\square$ Not Specified

Extended Data Fig. 2 | Distribution of the applied culture medium to prepare spheroids of the most reported cell line from non-breast tumors. Pie chart visualizing the proportion of culture media used to prepare A549 (lung), HCT116 (colorectal), HEPG2 (liver), PANC1 (pancreas), SKOV3 (ovarium) and U87MG (brain). 


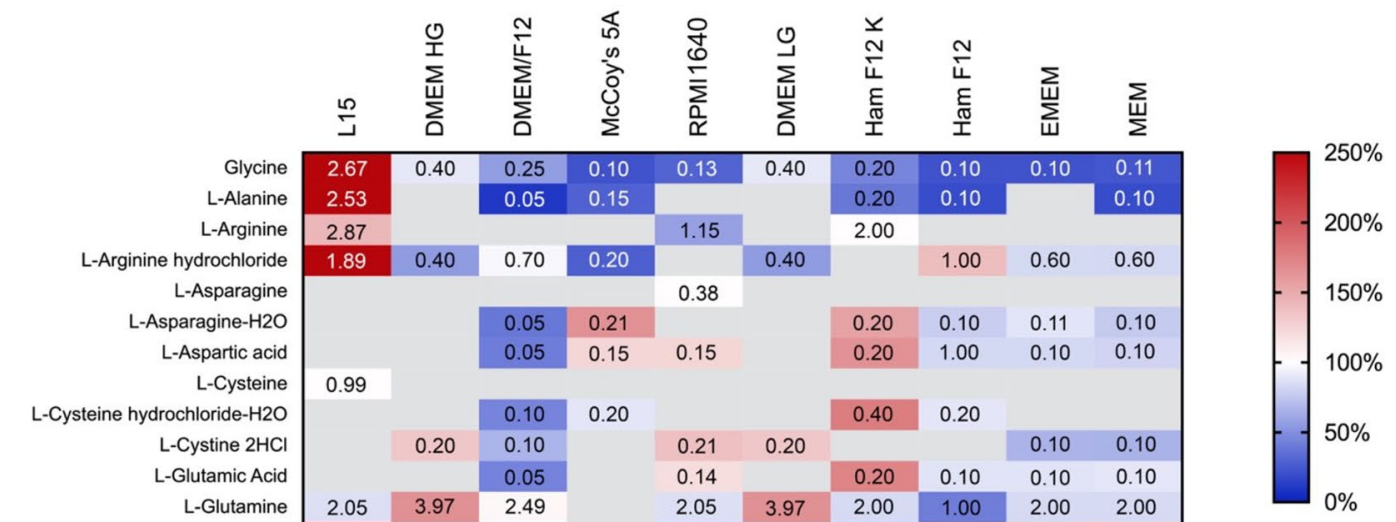

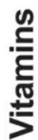

L-Histidine

L-Histidine hydrochloride-H2O

L-Hydroxyproline

L-Isoleucine

L-Leucine

L-Lysine

L-Lysine hydrochloride

L-Methionine

L-Phenylalanine

L-Proline

L-Serine

L-Threonine

L-Tryptophan

L-Tyrosine

L-Tyrosine disodium salt dihydrate

L-Valine

Ascorbic Acid*

Biotin

Choline chloride ${ }^{*}$

D-Calcium pantothenate*

Folic Acid*

Niacinamide*

Nicotinic Acid

Para-Aminobenzoic Acid ${ }^{*}$

Pyridoxal hydrochloride*

Pyridoxine hydrochloride*

Riboflavin*

Riboflavin 5'-phosphate $\mathrm{Na}^{*}$

Thiamine hydrochloride*

Thiamine monophosphate*

Vitamin B12*

i-Inositol ${ }^{\star}$

1.61

0.10

0.22

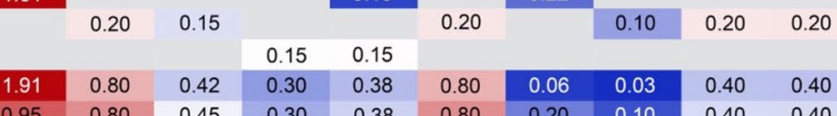

\begin{tabular}{|l|l|l|l|l|l|l|l|l|l|}
\hline 0.95 & 0.80 & 0.45 & 0.30 & 0.38 & 0.80 & 0.20 & 0.10 & 0.40 & 0.40 \\
\hline 0.51 & & & & & & & & & \\
\hline
\end{tabular}

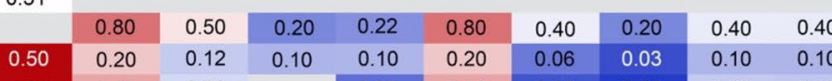

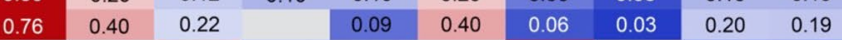

\begin{tabular}{|l|l|l|l|l|l|l|l|l|l|}
\hline & & 0.33 & 0.15 & 0.17 & & 0.60 & 0.30 & 0.10 & 0.10 \\
\hline 1.90 & 0.40 & 0.50 & 0.25 & 0.29 & 0.40 & 0.20 & 0.10 & 0.10 & 0.10 \\
\hline
\end{tabular}

\begin{tabular}{|l|l|l|l|l|l|l|l|ll|}
2.05 & 0.80 & 0.12 & 0.15 & 0.17 & 0.80 & 0.19 & 0.10 & 0.40 & 0.40 \\
\hline 0.10 & 0.08 & 0.18 & 0.02 & 0.02 & 0.08 & 0.02 & 0.01 & 0.05 & 0.05
\end{tabular}

\begin{tabular}{l|l|l|}
1.66 & 0.40 & 0.01
\end{tabular}

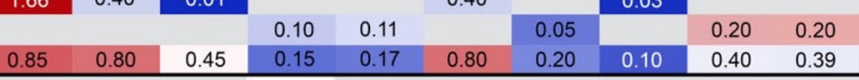

\begin{tabular}{|l|l|l|l|l|l|}
\hline & 2.84 & & & & \\
\hline & 0.01 & 0.82 & 0.82 & 0.29 & 0.03 \\
\hline
\end{tabular}

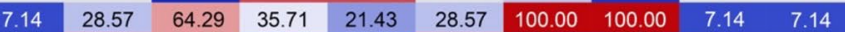

\begin{tabular}{|l|l|l|l|l|l|l|l|l|l|}
\hline 2.10 & 8.39 & 4.72 & 0.42 & 0.52 & 8.39 & 1.05 & 1.05 & 2.10 & 2.10 \\
\hline
\end{tabular}

\begin{tabular}{|l|l|l|l|l|l|l|l|l|l|}
2.27 & 9.07 & 6.01 & 22.68 & 2.27 & 9.07 & 2.95 & 2.95 & 2.27 & 2.27 \\
\hline
\end{tabular}

\begin{tabular}{|l|l|l|l|l|l|l|l|l|l|}
8.20 & 32.79 & 16.54 & 4.10 & 8.20 & 32.79 & 0.30 & 0.30 & 8.20 & 8.20 \\
\hline
\end{tabular}

4.09

$7.30 \quad 7.30$

\begin{tabular}{|l|c|c|c|c|c|c|c|c|c|}
\hline 4.85 & 19.42 & 9.85 & 4.85 & 4.85 & 19.42 & 0.29 & 0.29 & 4.86 & \\
\hline & 1.06 & 0.58 & 0.53 & 0.53 & 1.06 & 0.11 & 0.10 & 0.27 & 0.27 \\
\hline
\end{tabular}

0.21

2.26

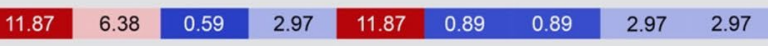

\begin{tabular}{|c|c|c|c|c|c|c|c|c|c|}
\hline & & 0.52 & 1.48 & 0.01 & \multicolumn{7}{c|}{1.03} & 1.03 & & \\
\hline 1.11 & 40.00 & 70.00 & 200.00 & 194.44 & 40.00 & 100.00 & 100.00 & 11.11 & 11.11 \\
\hline
\end{tabular}

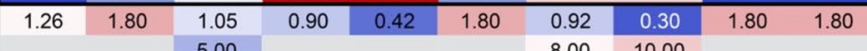

옹

롱

$\mathrm{Cu}^{2+}$

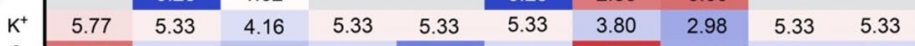

\begin{tabular}{|l|l|l|l|l|l|l|l|l|l|l|}
$\mathrm{Mg}^{2+}$ & 1.80 & 0.81 & 0.71 & 0.81 & 0.41 & 0.81 & 2.12 & 0.60 & 0.81 & 0.81 \\
\hline
\end{tabular}

\begin{tabular}{l|llllllllll}
$\mathrm{Na}^{+}$ & 139.27 & 155.31 & 150.66 & 141.34 & 132.89 & 155.31 & 160.83 & 146.02 & 136.11 & 136.11
\end{tabular} $\mathrm{Zn}^{2+*}$

$\mathrm{Cl}^{-}$

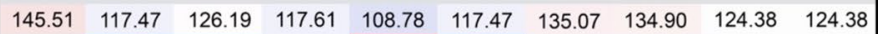

$\mathrm{NO}_{3}{ }^{-*}$

$\mathrm{HPO}_{4}{ }^{2-}$

$\mathrm{H}_{2} \mathrm{PO}_{4}^{-}$

$\mathrm{HCO}_{3}$

$\mathrm{SO}_{4}{ }^{2-}$

Bacto-Peptone

D+ Galactose

D-Glucose (Dextrose)

Glutathione (reduced)*

Hypoxanthine*

Linoleic Acid*

Lipoic Acid ${ }^{\star}$

Putrescine $2 \mathrm{HCl}^{*}$

Sodium Pyruvate

Thymidine $^{*}$

\begin{tabular}{|c|c|c|c|c|c|c|c|c|}
\hline 0.25 & 0.12 & & 423.73 & 0.25 & & & & \\
\hline 1.34 & 0.50 & & 5.63 & & 0.42 & 1.00 & & \\
\hline 0.92 & 0.46 & 0.92 & & 0.92 & 0.81 & & 1.01 & 1.01 \\
\hline 44.05 & 29.02 & 44.05 & 23.81 & 44.05 & 29.76 & 14.00 & 17.86 & 26.19 \\
\hline 0.81 & 0.41 & 0.81 & 0.41 & 0.81 & 1.60 & 0.01 & 0.81 & 0.81 \\
\hline & & 600.00 & & & & & & \\
\hline .00 & & & & & & & & \\
\hline 25.00 & 17.51 & 16.67 & 11.11 & 5.56 & 7.00 & 10.01 & 5.56 & 5.56 \\
\hline & & 1.63 & 3.26 & & & & & \\
\hline & 14.71 & & & & 31.70 & 29.41 & & \\
\hline & 0.15 & & & & & 0.30 & & \\
\hline & 0.51 & & & & 1.02 & 1.02 & & \\
\hline & 0.50 & & & & 1.99 & 1.00 & & \\
\hline 00 & 0.50 & & & 1.00 & 2.00 & 1.00 & 1.00 & \\
\hline & 1.45 & & & & 2.89 & 2.89 & & \\
\hline
\end{tabular}

Extended Data Fig. 3 | See next page for caption. 
Extended Data Fig. 3 | Comparison of the formulation of frequently reported culture media in spheroid research. Heat map showing the formulation of culture media types used in the empirical MISpherolD study, in addition with other media types frequently reported in spheroid research practices including L15, HamF12, HamF12K and McCoy's5A. Ranked according to decreasing nutrient richness from left to right. Nutrients that are not included in a medium type appear as a grey space in its corresponding column. Left column indicates nutrient type. Numerical value of nutrients in each medium is indicated in each cell. Values are concentrations, expressed in $\mathrm{mM}$, unless indicated ${ }^{*}=\mu \mathrm{M}, \#=\mathrm{nM}, \$=\mathrm{mg} / \mathrm{l}$. 
a

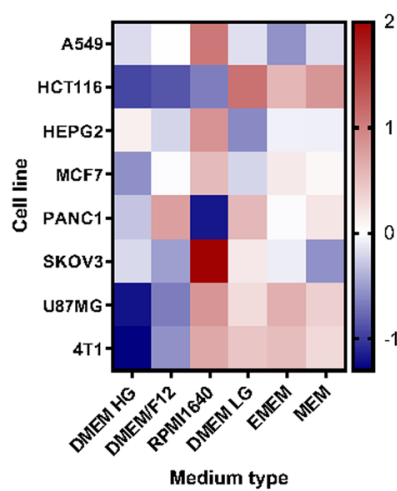

b
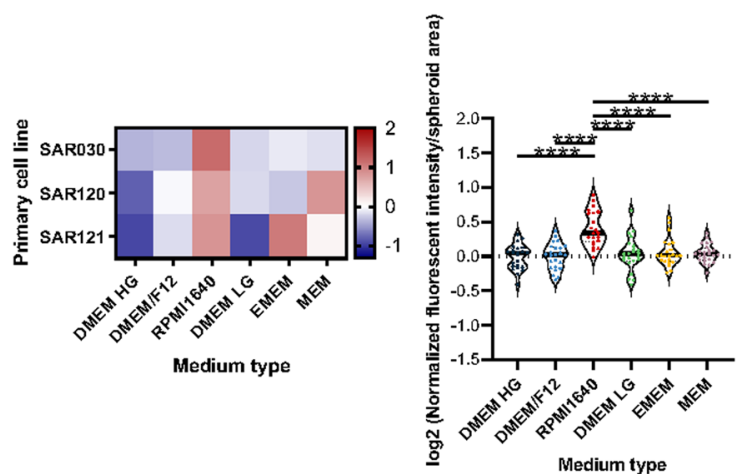

A549
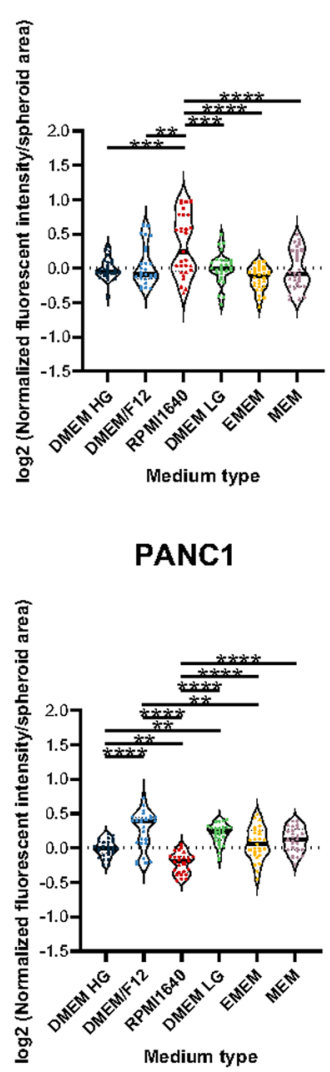

SAR030
PANC1

Extended Data Fig. 4 | Quantitative presentation of the impact of heterogeneity in culture medium on cell death in spheroids. Left, Z-score heatmaps and right, violin plots presenting the impact of six different media types on cell death in (a) 8 established cell lines and (b) 3 early passage, patient-derived sarcoma cultures. Biological replicates are indicated by a different symbol $(N \geq 3)$; each symbol is a technical replicate $(n=8)$. Y-axis represents log2-transformed data, all media types are normalized to DMEM HG. Horizontal bar indicates median. Statistical significance between the groups was determined with a one-way ANOVA and Tukey's multiple comparison test. ${ }^{\star \star} p<0.01,{ }^{\star \star *} p<0.001,{ }^{\star \star \star *} p<0.0001$. Colors in violin plots present media type as in Fig. 3a; media types are ranked from higher nutrient (left) to lower nutrient (right) richness.
HEPG2

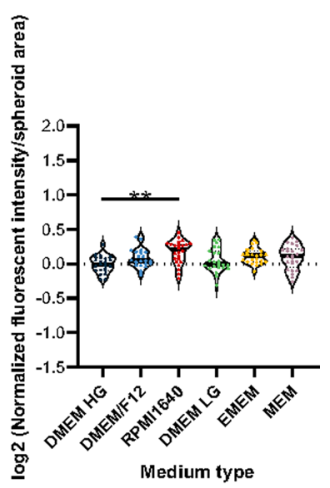

$4 \mathrm{T1}$

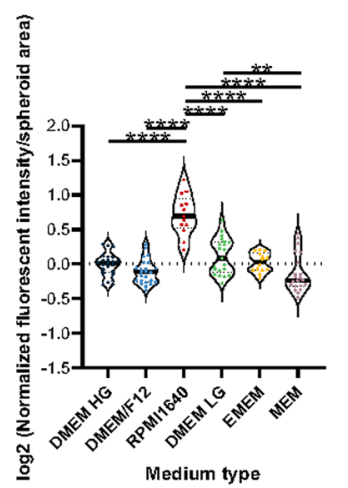

SAR120
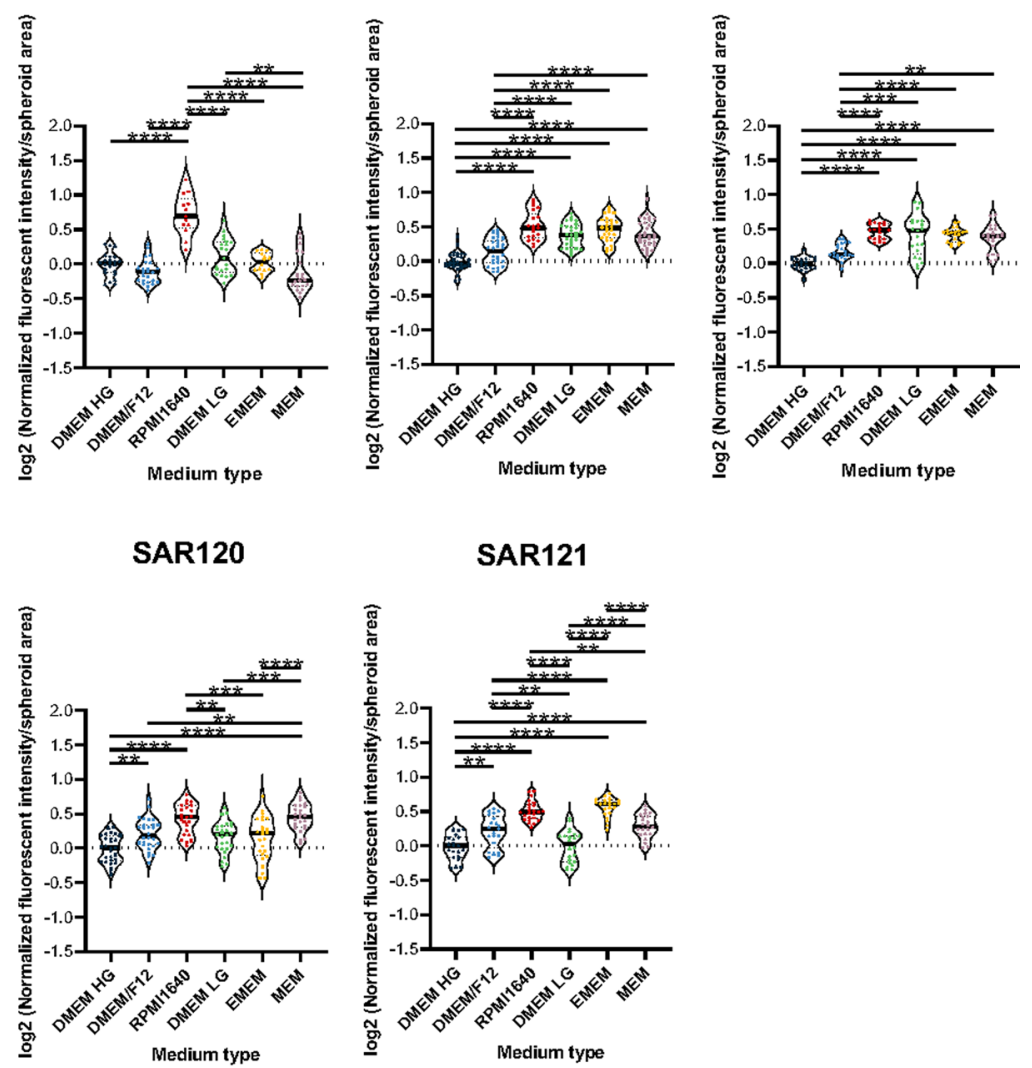

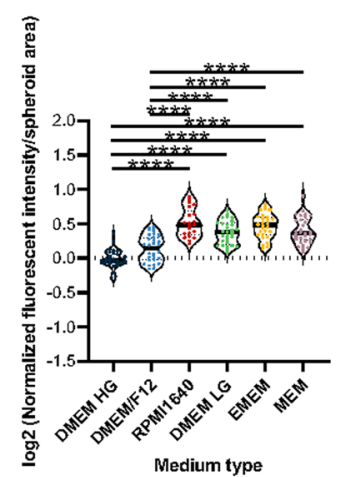

SAR121

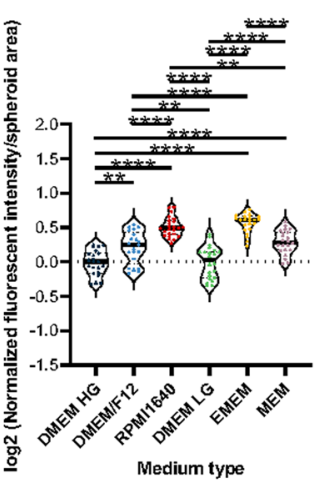




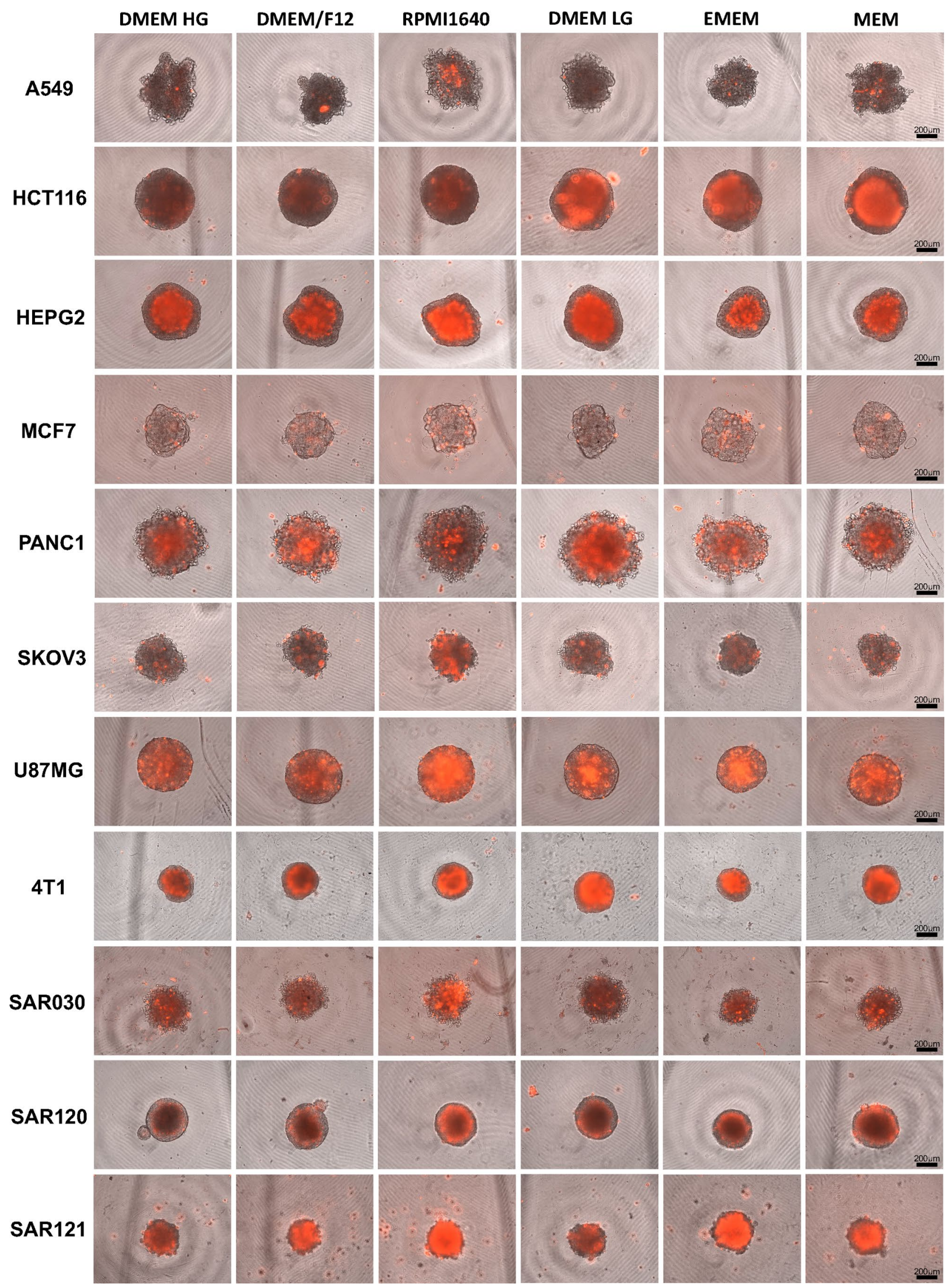

Extended Data Fig. 5 | See next page for caption. 
Extended Data Fig. 5 | Image presentation of cell death, circularity and size in spheroids cultured in six different media types. Representative microscopy images show ethidium homodimer I stained (red if cell is dead) spheroids of all evaluated cell lines and patient-derived sarcoma cells (cell types indicated on the left) cultured in different media types (indicated on the top) (scale bars $200 \mu \mathrm{m}$ ). Each experiment was repeated independently at least 3 times with 8 technical replicates per experiment, with similar results. Media types are ranked from higher nutrient (left) to lower nutrient (right) richness. Intense staining in the spheroid center is indicative of necrotic core. Next to cell death, images give an indication of circularity and size. 
a

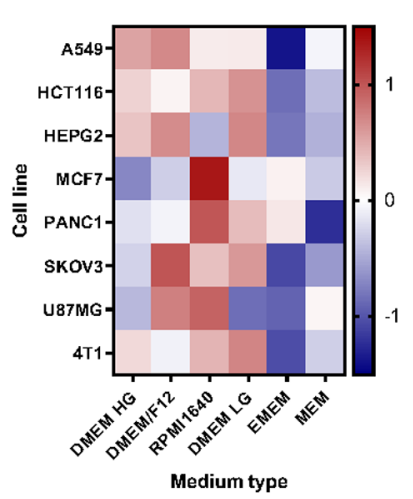

b

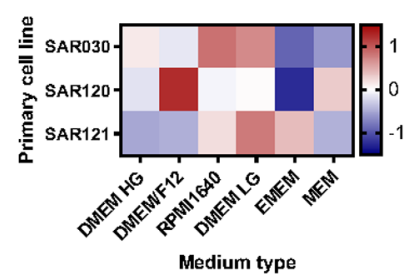

A549

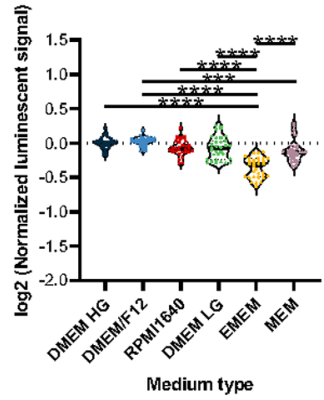

PANC1

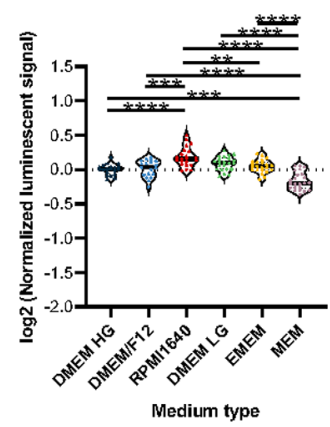

SAR030

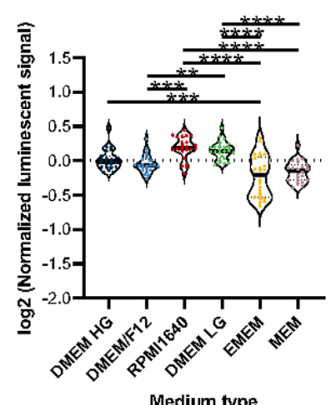

HCT116

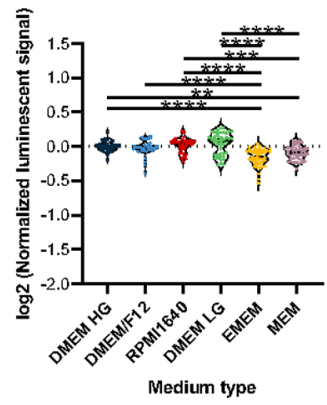

SKOV3

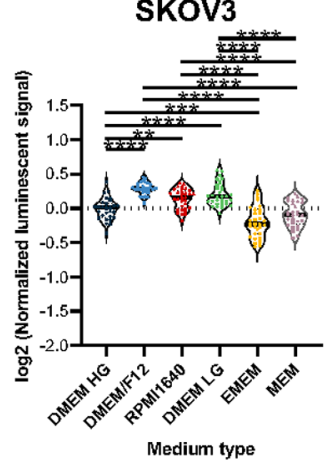

SAR120

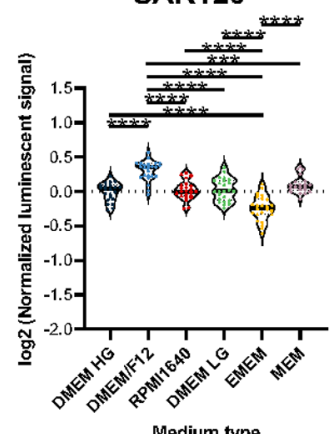

HEPG2

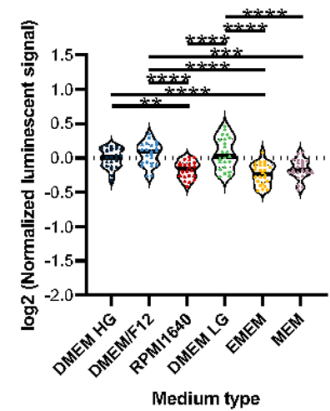

U87MG

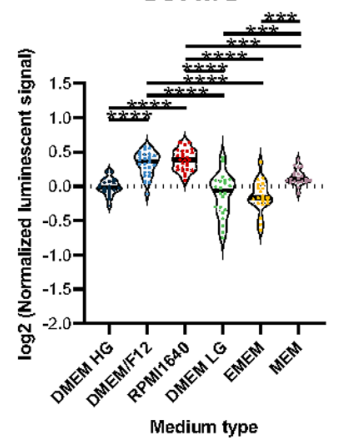

SAR121

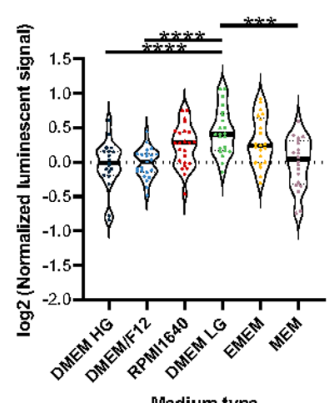

MCF7

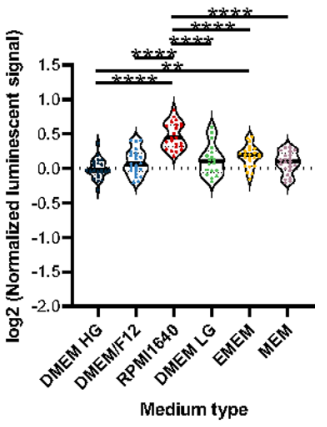

4T1

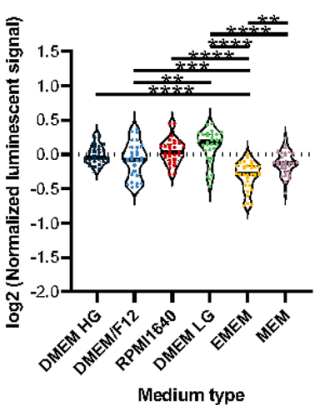

Medium type

Extended Data Fig. 6 | Quantitative presentation of the impact of heterogeneity in culture medium on ATP content in spheroids. Left, Z-score heatmaps and right, violin plots presenting the impact of six different media types on ATP content in (a) 8 established cell lines and (b) 3 early passage, patient-derived sarcoma cultures. Biological replicates are indicated by a different symbol $(N \geq 3)$; each symbol is a technical replicate $(n=8)$. $Y$-axis represents log2-transformed data, all media types are normalized to DMEM HG. Horizontal bar indicates median. Statistical significance between the groups was determined with a one-way ANOVA and Tukey's multiple comparison test. ${ }^{\star \star} p<0.01,{ }^{\star \star \star} p<0.001,{ }^{\star \star \star \star} p<0.0001$. Colors in violin plots present media type as in Fig. 3a; media types are ranked from higher nutrient (left) to lower nutrient (right) richness. 
RESOURCE

NATURE METHODS

a

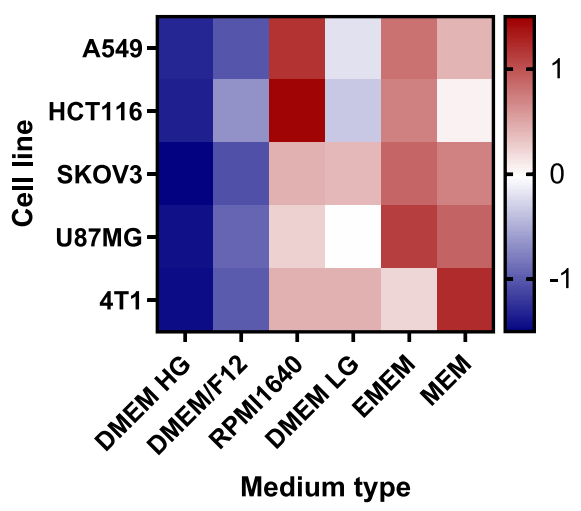

b

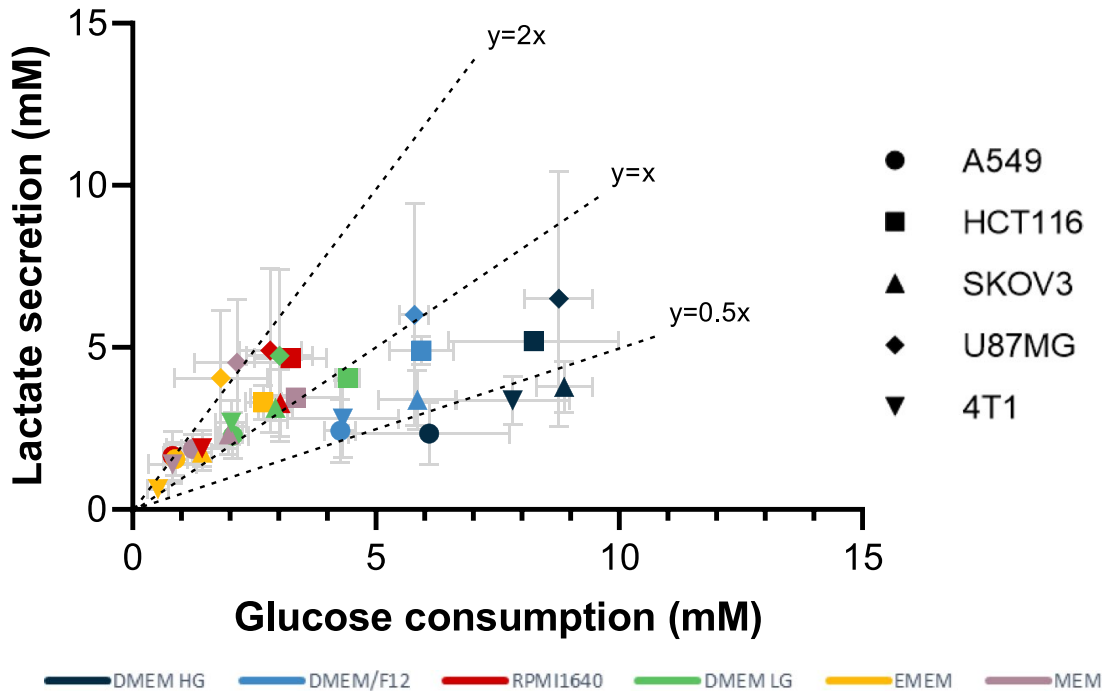

Extended Data Fig. 7 | Quantitative presentation of the impact of heterogeneity in culture medium on ratio of glucose uptake to lactate secretion in spheroids. Left, Z-score heatmap and right, scatter diagram presenting the impact of six different media types on ratio of glucose (G) uptake to lactate ( $L$ ) secretion ( $L / G$ ratio) in 5 established cell lines $(N \geq 2, n=4)$. Indicative trendlines are presented in the scatter diagram with a dotted line (higher slope means a higher ratio of lactate secretion to glucose uptake (for example $y=2 x$; for the consumption of one glucose molecule 2 lactate molecules are produced)). Error bars indicate SD. Colors in scatter diagram present media type as in Fig. Ba; media types are ranked from higher nutrient (left) to lower nutrient (right) richness.

NATURE METHODS | www.nature.com/naturemethods 
a

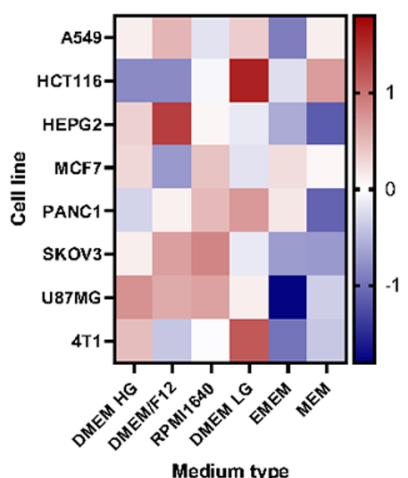

b

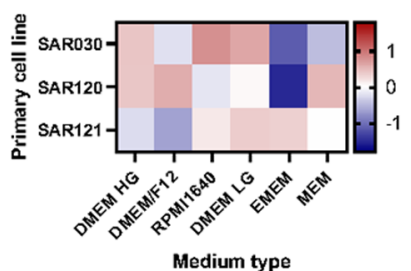

Medium type
A549

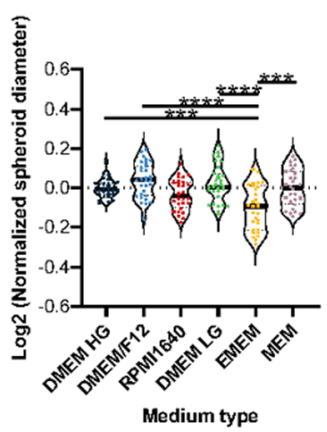

PANC1

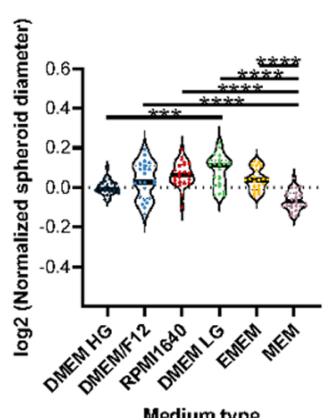

SAR030

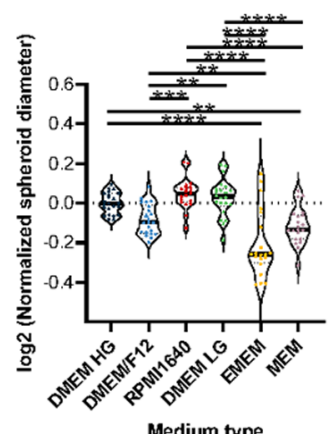

HCT116

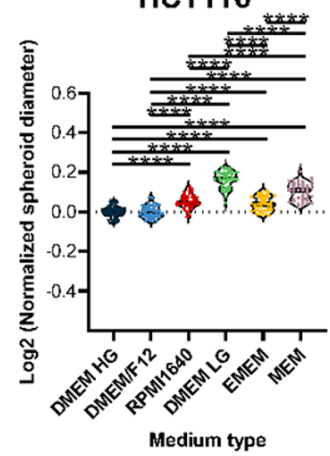

SKOV3

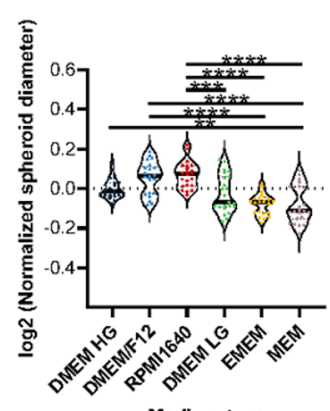

Medium type

SAR120

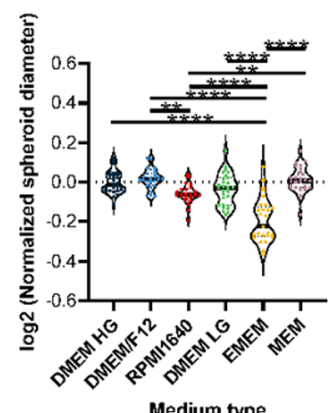

Medium type
HEPG2

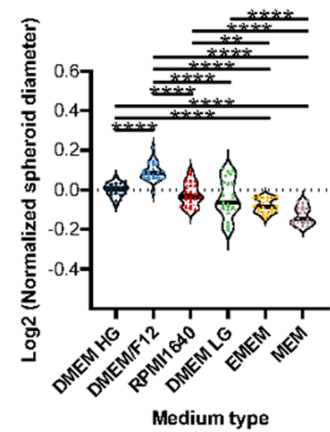

U87MG

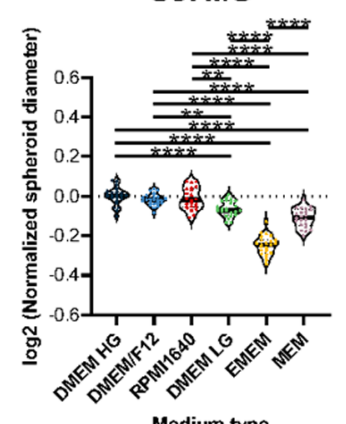

Medium type

SAR121

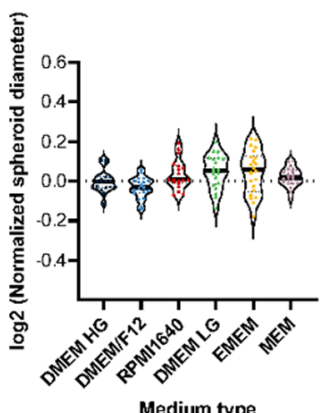

Extended Data Fig. 8 | Quantitative presentation of the impact of heterogeneity in culture medium on size of spheroids. Left, Z-score heatmaps and right, violin plots presenting the impact of six different media types on spheroid size difference in (a) 8 established cell lines and (b) 3 early passage, patient-derived sarcoma cultures. Biological replicates are indicated by a different symbol $(N \geq 3)$; each symbol is a technical replicate $(n=8)$. $Y$-axis represents log2-transformed changes in size, all media types are normalized to DMEM HG. Horizontal bar indicates median. Statistical significance between the groups was determined with a one-way ANOVA and Tukey's multiple comparison test. ${ }^{\star \star} p<0.01,{ }^{\star \star \star} p<0.001,{ }^{\star \star \star \star} p<0.0001$. Colors in violin plots present media type as in Fig. 3a; media types are ranked from higher nutrient (left) to lower nutrient (right) richness. 
a

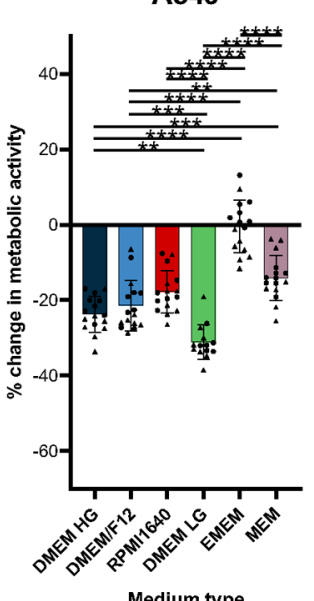

b

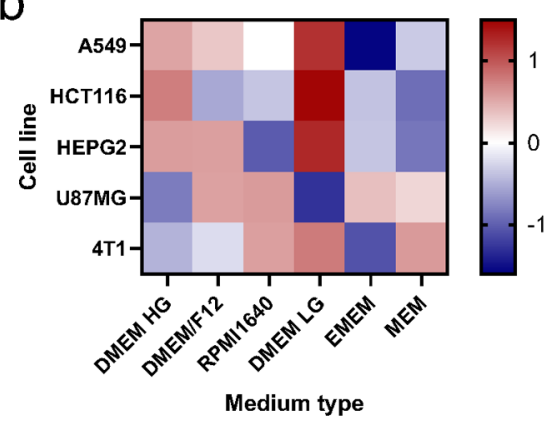

C A549

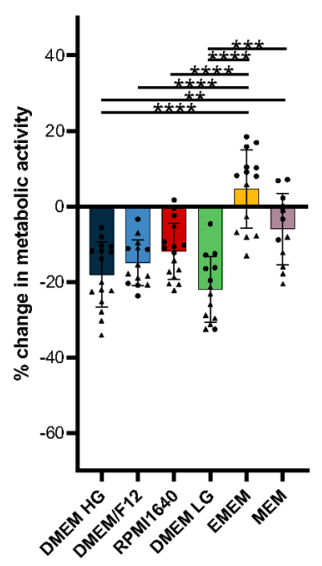

Medium type

d
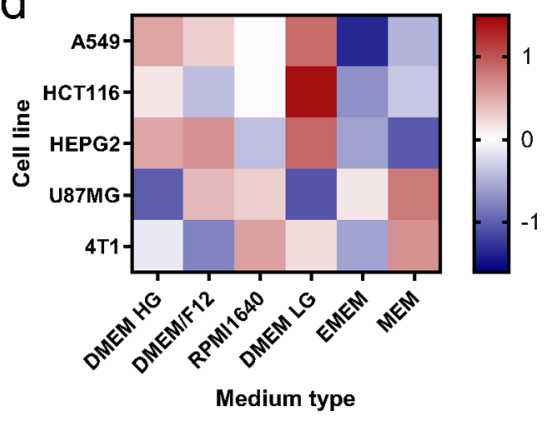

HCT116

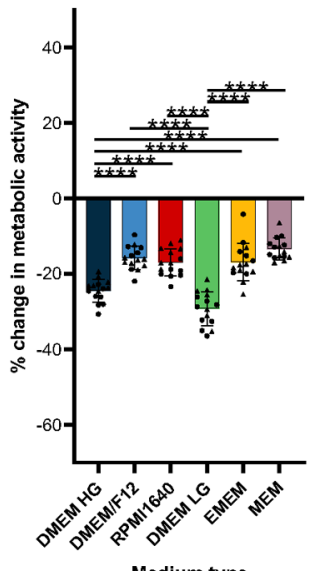

Medium type
HEPG2

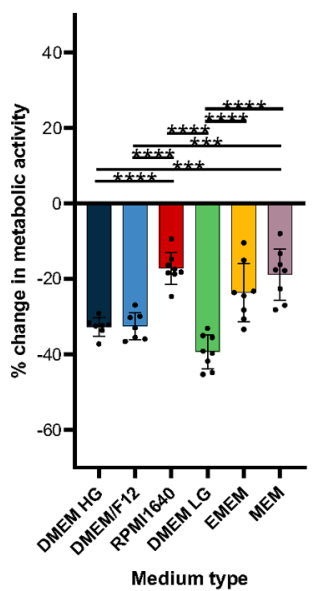

U87MG

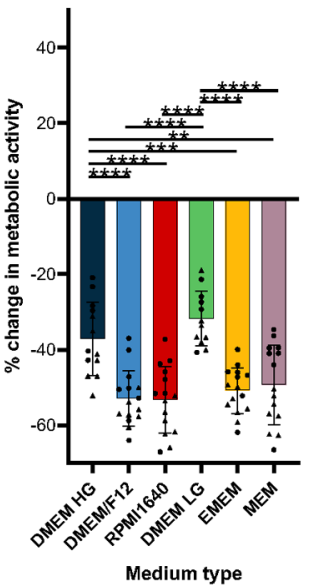

$4 \mathrm{T1}$

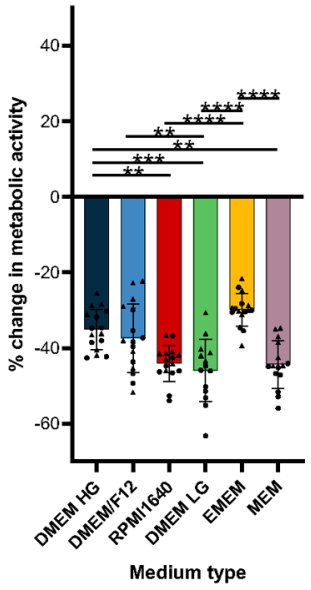

HEPG2

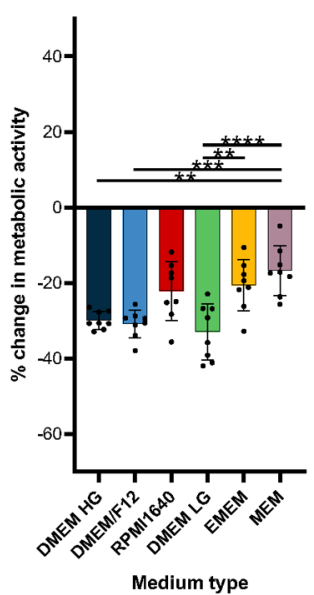

U87MG

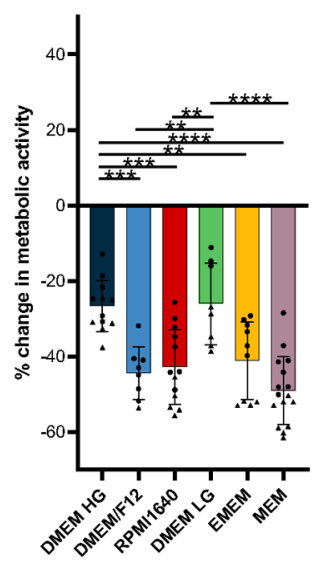

Medium type
4T1

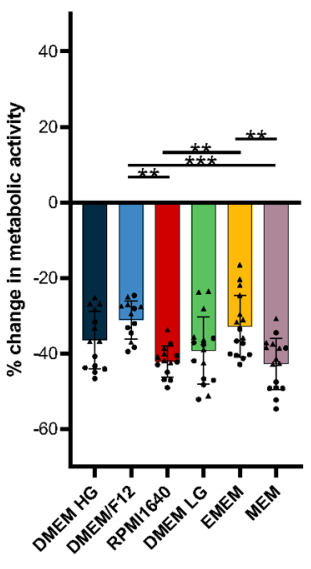

Medium type

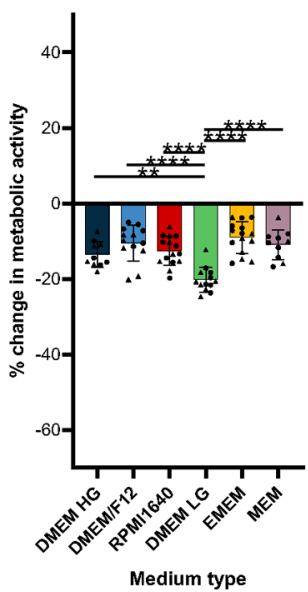

Extended Data Fig. 9 | See next page for caption. 
Extended Data Fig. 9 | Response to radiotherapy of spheroids cultured in diverse media types. Bar plots (a and $\mathbf{c}$ ); and Z-score heatmaps (b and $\mathbf{d}$ ) respectively indicate the impact of a single ( $a$ and b) $20 \mathrm{~Gy}$ and ( $\mathrm{c}$ and d) $10 \mathrm{~Gy}$ fraction on ATP content of spheroids of indicated cell lines cultured in different media types. Biological replicates are indicated by a different symbol $(N=2$; except HEPG2 $N=1)$; each symbol is a technical replicate $(n=8)$. Statistical significance between the groups was determined with a one-way ANOVA and Tukey's multiple comparison test. ${ }^{\star \star} p<0.01,{ }^{\star \star \star} p<0.001$,

${ }^{\star \star \star \star} p<0.0001$. Horizontal bar indicates median, error bars indicate SD. Colors in bar plots present media type as in Fig. 3a; media types are ranked from higher nutrient (left) to lower nutrient (right) richness. 
a

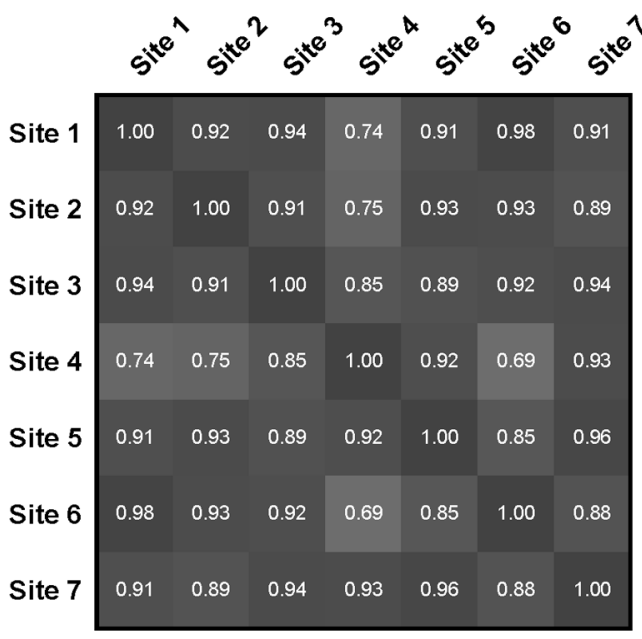

b

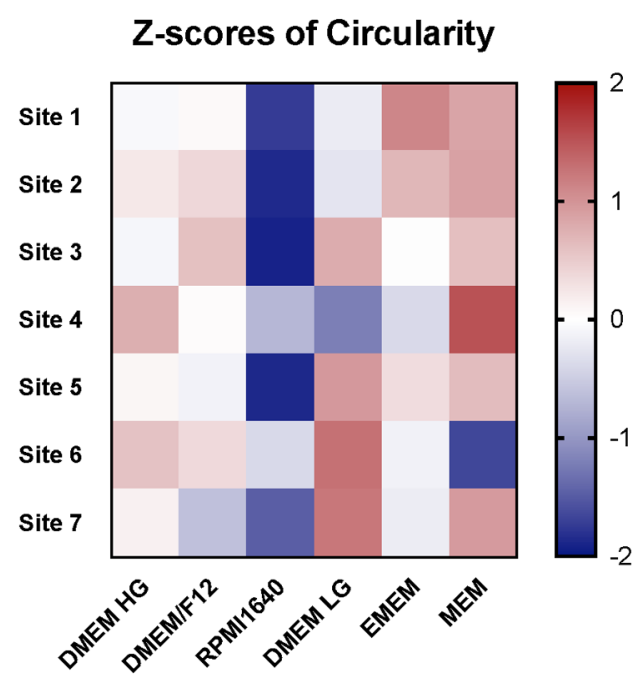

Medium Type

C

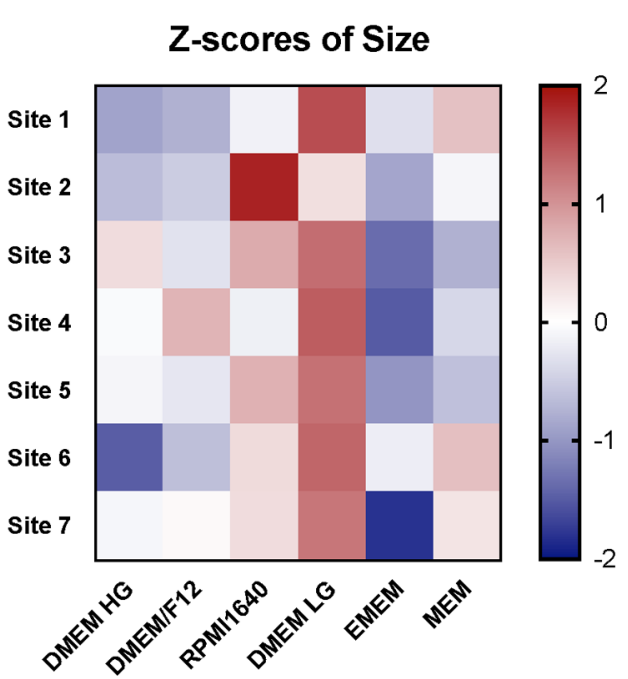

Medium Type
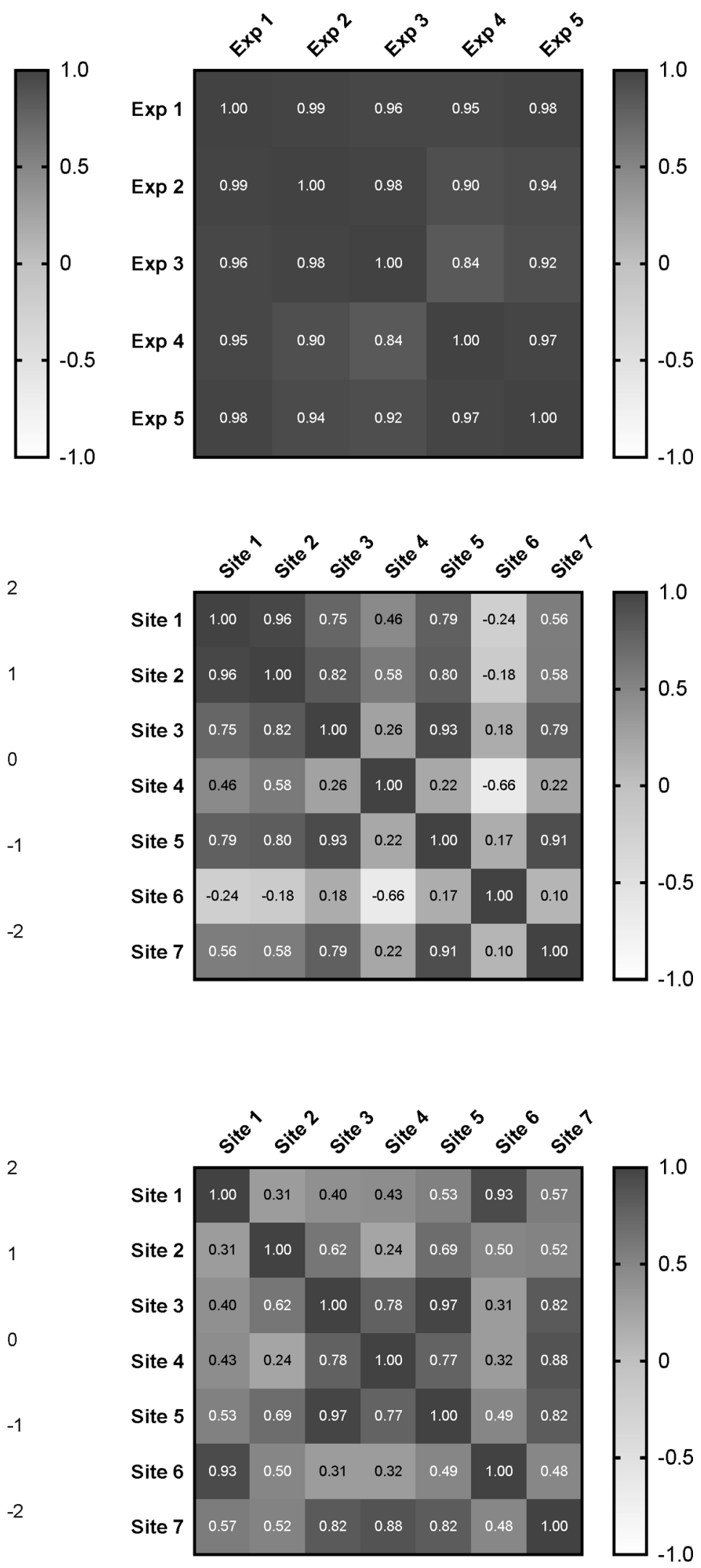

Extended Data Fig. 10 | See next page for caption. 
Extended Data Fig. 10 | Evaluation of the metrics cell death, circularity and size in an interlaboratory study. (a) Correlation matrix of cell death ranking of HCT116 spheroids cultured in six different media types between different participating sites in the interlaboratory study (left) and between biological replicates in the study-initiating laboratory (right). In (b) and (c), Z-score heatmaps (left) and correlation matrices (right) present the impact of spheroid circularity (b) and size (c) of HCT116 spheroids cultured in six different media types evaluated across seven different sites in an interlaboratory setting. Media types in Z-score heatmaps are ranked from higher nutrient (left) to lower nutrient (right) richness. The median Spearman correlation across the entire dataset is respectively 0.56 for circularity and 0.57 for size. The larger spread in correlation coefficient of circularity across laboratories is due to subtle absolute differences with most probably limited biological significance. 


\section{Reporting Summary}

Nature Research wishes to improve the reproducibility of the work that we publish. This form provides structure for consistency and transparency in reporting. For further information on Nature Research policies, see our Editorial Policies and the Editorial Policy Checklist.

\section{Statistics}

For all statistical analyses, confirm that the following items are present in the figure legend, table legend, main text, or Methods section.

$\mathrm{n} / \mathrm{a}$ Confirmed

$\bigotimes$ The exact sample size $(n)$ for each experimental group/condition, given as a discrete number and unit of measurement

$\bigotimes$ A statement on whether measurements were taken from distinct samples or whether the same sample was measured repeatedly

The statistical test(s) used AND whether they are one- or two-sided

Only common tests should be described solely by name; describe more complex techniques in the Methods section.

Х $\square$ A description of all covariates tested

Х A description of any assumptions or corrections, such as tests of normality and adjustment for multiple comparisons

$\triangle$ A full description of the statistical parameters including central tendency (e.g. means) or other basic estimates (e.g. regression coefficient)

AND variation (e.g. standard deviation) or associated estimates of uncertainty (e.g. confidence intervals)

For null hypothesis testing, the test statistic (e.g. $F, t, r$ ) with confidence intervals, effect sizes, degrees of freedom and $P$ value noted Give $P$ values as exact values whenever suitable.

Х $\square$ For Bayesian analysis, information on the choice of priors and Markov chain Monte Carlo settings

Х $\square$ For hierarchical and complex designs, identification of the appropriate level for tests and full reporting of outcomes

$\square \bigotimes$ Estimates of effect sizes (e.g. Cohen's $d$, Pearson's $r$ ), indicating how they were calculated

\section{Our web collection on statistics for biologists contains articles on many of the points above.}

\section{Software and code}

Policy information about availability of computer code

Data collection NextSeq 500 software v.4.0.1; Gen5 Data analysis software v3.08.01; AxioVision Rel. 4.8

Data analysis DESeq2 v1.30.1; GSEA software v4.1.0; PAST4.03; AnaSP v1.4; Excel v.2106; Graphpad Prism v8.4.3; ImageJ v1.52v.

For manuscripts utilizing custom algorithms or software that are central to the research but not yet described in published literature, software must be made available to editors and

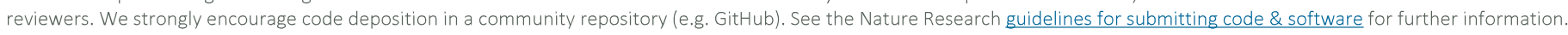

\section{Data}

Policy information about availability of data

All manuscripts must include a data availability statement. This statement should provide the following information, where applicable:

- Accession codes, unique identifiers, or web links for publicly available datasets

- A list of figures that have associated raw data

- A description of any restrictions on data availability

RNA sequencing data generated during the current study will be available in the ArrayExpress database (www.ebi.ac.uk/arrayexpress) under accession number EMTAB-10862.

The source data underlying Figure 4 and 5, Extended Figures 4-10, Supplementary Figures 9-13 and Table 6 are provided as a source data file. All literature study data are available through the MISpherolD knowledgebase. 
Please select the one below that is the best fit for your research. If you are not sure, read the appropriate sections before making your selection.

\ Life sciences

Behavioural \& social sciences

Ecological, evolutionary \& environmental sciences

For a reference copy of the document with all sections, see nature.com/documents/nr-reporting-summary-flat.pdf

\section{Life sciences study design}

All studies must disclose on these points even when the disclosure is negative.

Sample size Dependent on the metric investigated, four (transcriptional variation and ratio of glucose uptake to lactate secretion) to minimally eight (all other) technical replicates are included in each experiment, reproducibility was further demonstrated with multiple biological replicates. Sample size are indicated in the legends of all relevant figures and in the Material\&Methods section.

Sample sizes were justified a posteriori since differences were highly statistical significant (p-values lower than 0.01 are shown).

Data exclusions Data points were first excluded manually (e.g. spheroids containing a dust particle when measuring dead signal, spheroids that were lost when pipetting to a white plate for measuring metabolic activity with cellTiter-glo3D, etc.). Afterwards, outliers were calculated via the ROUT method $(Q=1 \%)$ in the Graphpad Prism software.

$\begin{array}{lll}\text { Replication } & \text { Reproducibility of the experimental findings was verified in at least } 3 \text { biological replicates for most spheroid metrics and an interlaboratory }\end{array}$ study between 7 sites investigates the impact of culture medium type on three spheroid metrics. All attempts at replication were succesful.

Randomization No randomization was included as this was not relevant to the study design.

\begin{tabular}{l|l} 
Blinding & The study was not blinded since dead stainings were often distinctly observable between groups (dead staining assay) or the results were
\end{tabular} obtained computationally (all other assays).

\section{Reporting for specific materials, systems and methods}

We require information from authors about some types of materials, experimental systems and methods used in many studies. Here, indicate whether each material, system or method listed is relevant to your study. If you are not sure if a list item applies to your research, read the appropriate section before selecting a response.

\begin{tabular}{l|l} 
Materials \& experimental systems \\
\hline$n / a$ & Involved in the study \\
$\square$ & $\square$ Antibodies \\
$\square$ & $\square$ Eukaryotic cell lines \\
$\square$ & $\square$ Animals and other organisms \\
$\square$ & $\square$ Clinical data \\
$\square$ & $\square$ Dual use research of concern
\end{tabular}

Methods

$\mathrm{n} / \mathrm{a}$ Involved in the study

X $\square$ ChIP-seq

Х $\square$ Flow cytometry

\ $\square$ MRI-based neuroimaging

\section{Eukaryotic cell lines}

Policy information about cell lines

Cell line source(s)

The following cell lines: A549, HCT116, HEPG2, MCF7, PANC1, SKOV3, U87MG and 4T1 were purchased from the American Type Culture Collection. SAR030, SAR120 and SAR121 are primary cell cultures established in the lab.

Authentication

Mycoplasma contamination

Commonly misidentified lines (See ICLAC register)
All human cell lines were authenticated using a 21-Marker STR Profile test (Eurofins, Luxembourg).

All cell lines were monthly tested for mycoplasma contamination using the Mycoalert Mycoplasma Detection Kit and tested negative.

No commonly misidentified cell lines were used in the study. 